TRANSACTIONS OF THE

AMERICAN MATHEMATICAL SOCIETY

Volume 351, Number 11, Pages 4629-4656

S 0002-9947(99)02414-9

Article electronically published on April 12, 1999

\title{
ADMISSIBILITY OF WEIGHTS ON NON-NORMED $*$-ALGEBRAS
}

\author{
S. J. BHATT, A. INOUE, AND H. OGI
}

\begin{abstract}
The notion of weights on (topological) *-algebras is defined and studied. The primary purpose is to define the notions of admissibility and approximate admissibility of weights, and to investigate when a weight is admissible or approximately admissible. The results obtained are applied to vector weights and tracial weight on unbounded operator algebras, as well as to weights on smooth subalgebras of a $\mathrm{C}^{*}$-algebra.
\end{abstract}

\section{INTRODUCTION}

Non-commutative integration deals with positive linear functionals and weights on a non-commutative $*$-algebra $\mathcal{A}$ as well as the $*$-representations of $\mathcal{A}$ induced by them. When $\mathcal{A}$ is a von Neumann algebra or a $\mathrm{C}^{*}$-algebra, it has become an essential part of the theory. In fact, much of the theory of these algebras can be regarded as a non-commutative analogue of integration theory, the role of finite (resp. infinite) measure being played by a positive linear functional (resp. weight). Weights have also appeared naturally in unbounded operator algebras in the context of unbounded Tomita-Takesaki theory [16], [17], as well as in quantum physics [3]. In the framework of unbounded representation theory [25], this leads to weights on $\mathcal{A}$. Though positive linear functionals and the GNS representations of $\mathcal{A}$ induced by them have been greatly analyzed, no such attempt seems to have been made for theory of weights. The purpose of the present paper is to initiate the study of weights on abstract $*$-algebras. The paper is roughly devided into two parts. Part I constitutes Sections 2 - 5, in which admissibility and approximate (and strict in-) admissibility (a phenomena not found in $\mathrm{C}^{*}$-algebras) of weights are investigated. Section 6 constitutes Part II, in which the abstract results of Part I are applied to a number of concrete examples.

Let $\mathcal{P}(\mathcal{A})$ denote the positive cone of $\mathcal{A}$. A weight on $\mathcal{A}$ is a map $\varphi: \mathcal{P}(\mathcal{A}) \rightarrow$ $\mathbb{R}_{+} \cup\{\infty\}$ such that (i) $\varphi(x+y)=\varphi(x)+\varphi(y)$, and (ii) $\varphi(\lambda x)=\lambda \varphi(x)$ for all $x, y$ in $\mathcal{P}(\mathcal{A})$ and all $\lambda \geq 0$. Unlike $\mathrm{C}^{*}$-algebras, $\mathfrak{N}_{\varphi}^{0} \equiv\left\{x \in \mathcal{A} ; \varphi\left(x^{*} x\right)<\infty\right\}$ need not be a left ideal of $\mathcal{A}$. However, $\mathfrak{N}_{\varphi} \equiv\left\{x \in \mathcal{A}: \varphi\left(x^{*} a^{*} a x\right)<\infty, \forall a \in \mathcal{A}\right\}$ is a left ideal, and $\varphi \Gamma_{\mathcal{P}\left(\mathfrak{N}_{\varphi}\right)}=\psi$ is a quasi-weight in the sense that $\psi$ is defined on the positive cone $\mathcal{P}\left(\mathfrak{N}_{\varphi}\right)$ of the left ideal $\mathfrak{N}_{\varphi}$ such that $\psi: \mathcal{P}\left(\mathfrak{N}_{\varphi}\right) \rightarrow \mathbb{R}_{+}$ satisfies (i) and (ii). The construction of the GNS-representation $\pi_{\varphi}$ of $\mathcal{A}$ using a quasi-weight $\varphi$ on $\mathcal{P}\left(\mathfrak{N}_{\varphi}\right)$ summarized in Section 2 . In general, $\pi_{\varphi}$ maps $\mathcal{A}$ into unbounded operators in a Hilbert space. We call $\varphi$ admissible if $\pi_{\varphi}$ is a bounded operator representation. Such quasi-weights are characterized in Section

Received by the editors February 23, 1997.

1991 Mathematics Subject Classification. Primary 46K10, 47D40. 
3 , and sufficient conditions for admissibility are investigated. Next we consider the question: When is every (quasi-) weight on $\mathcal{A}$ admissible? In fact, the notion of quasi-weights introduces a kind of boundedness structure in $\mathcal{A}$. This is investigated and exhibited in several class of $*$-algebras like Banach $*$-algebras, the $\mathrm{BG}^{*}$-algebras of Palmer [19], locally convex *-algebras, in particular, generalized $\mathrm{B}^{*}$-algebras [2], [12], and pro-C*-algebras [21]. It is shown in Theorem 3.12 that if $\mathcal{A}$ is a pseudocomplete locally convex $*$-algebra in which $\mathcal{A}=\mathcal{A}_{0}$ (the Allan bounded elements of $\mathcal{A}[1]$ ), then every quasi-weight on $\mathcal{A}$ is admissible. In Section 4 , conditions ensuring $\mathcal{A}=\mathcal{A}_{0}$ are investigated. A (quasi-) weight $\varphi$ on a $*$-algebra $\mathcal{A}$ is approximately admissible if $\varphi=\lim _{\alpha} \varphi_{\alpha}$ for a net $\left\{\varphi_{\alpha}\right\}$ of admissible (quasi-) weights. Such (quasi-) weights are investigated in Section 5. It is shown in Theorem 5.6 that if $\varphi$ is a regular quasi-weight such that $\pi_{\varphi}$ is self-adjoint, then $\varphi$ is approximately admissible iff $\pi_{\varphi}$ is a direct sum of bounded operator representations. In Section 6 , we discuss the following, exhibiting weights in a number of interesting non- $\mathrm{C}^{*}$ algebra situations.

(1) Weight analogue of the Bochner-Weil-Raikov theorem: An integral representation of quasi-weights on a commutative Banach $*$-algebra is obtained. This provides weight analogue of the Bochner-Weil-Raikov Theorem, and is used to define the Fourier transform of unbounded measures on a locally compact abelian group.

(2) Integration algebras, measures and tracial weights: $\mathrm{A} *$-algebra $\mathcal{A}$ with a faithful (quasi-) weight $\varphi$ constitutes what we call an integration algebra. In the framework of classical measure theory, admissibility of $\varphi$ corresponds to finiteness of measure, whereas approximate admissibility corresponds roughly to $\sigma$-finiteness. Let $\mathcal{M}$ be an $\mathrm{O}^{*}$-algebra (unbounded operator algebra) defined on a dense subspace $\mathcal{D}$ of a Hilbert space $\mathcal{H}$. For $\Omega$ a positive operator, $\varphi\left(X^{\dagger} X\right)=\operatorname{tr}\left(X^{\dagger} X\right)$, $\varphi_{\Omega}\left(X^{\dagger} X\right)=\operatorname{tr}\left(X^{\dagger} X \Omega^{2}\right) \quad\left(X \in \mathcal{M}, X^{\dagger}=X^{*}\left\lceil_{\mathcal{D}}\right)\right.$ define quasi-weights in $\mathcal{M}$. Conditions on $\mathcal{M}$ are investigated under which $\varphi$ and $\varphi_{\Omega}$ are admissible (resp. approximately admissible, strictly inadmissible). Such quasi-weights $\varphi_{\Omega}$ appear in several cases of interest in quantum theory.

(3) Vector (quasi-) weights defined by $*$-representations: Let $(\pi, \mathcal{D}(\pi), \mathcal{H})$ be a $*$-representation of a $*$-algebra $\mathcal{A}$ into linear operators all defined on a dense invariant subspace $\mathcal{D}(\pi)$ of a Hilbert space $\mathcal{H}$. A vector $\xi \in \mathcal{H} \backslash \mathcal{D}\left(\pi^{*}\right)$ defines a vector quasi-weight $f_{\xi}$, which is not necessarily a positive functional, as follows. Take

$$
\begin{aligned}
& \mathfrak{N}_{f_{\xi}}=\left\{x \in \mathcal{A}: \xi \in \mathcal{D}\left(\pi\left(x^{*}\right)^{*}\right), \pi\left(x^{*}\right)^{*} \xi \in \mathcal{D}(\pi)\right\} . \\
& f_{\xi}\left(x^{*} x\right)=\left\|\pi\left(x^{*}\right)^{*} \xi\right\|^{2}, \quad x \in \mathfrak{N}_{f_{\xi}} .
\end{aligned}
$$

If $\mathcal{A}$ is the $*$-algebra of polynomially dominated measurable functions on $\mathbb{R}_{+}$and if $\pi$ is the $*$-representation defined by the spectral theorem of a positive self-adjoint operator $h$, then $f_{\xi}$ is approximately admissible, and it is admissible iff $h$ is bounded. On the other hand, for the Schrödinger representation of the CCR algebra, $f_{\xi}$ is far away from admissibility.

(4) Point evalutions: Let $C(X)$ be the algebra of all continuous complex functions on $X$, and let $C_{b}(X)=\{f \in C(X): f$ is bounded $\}$. Let $\nu X$ and $\beta X$ be the Hewitt real compactification and the Stone-Čech compactification of $X$, respectively. Every $x \in \beta X \backslash \nu X$ defines an admissible weight in a $*$-subalgebra $\mathcal{A}$ of $C(X)$ containing $C_{b}(X)$. 
(5) Weights on smooth subalgebras of a $C^{*}$-algebra: Let $\mathcal{B}$ be a smooth *subalgebra of a $\mathrm{C}^{*}$-algebra $\mathcal{A}$. It is shown that every quasi-weight on $\mathcal{B}$ is admissible, and under an additional assumption, it extends to a quasi-weight on $\mathcal{A}$.

(6) Quasi-weights in non-commutative geometry: In the framework of the Connes theory of non-commutative geometry, let $\Omega(\mathcal{A})$ be the $*$-algebra of non-commutative differential forms over a $*$-algebra $\mathcal{A}$. Let $(\pi, \mathcal{H}, \mathcal{D})$ be a K-cycle, and let $\operatorname{Tr}_{\omega}$ denote the Dixmier trace on $B(\mathcal{H})$. Then $\tau_{\omega}\left(x^{*} x\right)=\operatorname{Tr}_{\omega}\left(\pi(x)^{*} \pi(x)\right) \quad(x \in \Omega(\mathcal{A}))$ defines an admissible quasi-weight on $\Omega(\mathcal{A})$. The $d$-dimensional volume integral $a \rightarrow f \pi(a)|D|^{-d}$ gives an admissible quasi-weight on $\mathcal{A}$, which is finite iff the $\mathrm{K}$-cycle is $d^{+}$-summable. The map $\psi\left(x^{*} x\right)=\operatorname{Lim}_{\varepsilon \rightarrow 0} \operatorname{tr}\left(\pi\left(x^{*} x\right) e^{-\varepsilon D^{2}}\right.$ ) (limit in suitable Cesáro mean) gives an admisssible quasi-weight in $\Omega(\mathcal{A})$, and is finite iff the K-cycle is $\theta$-summable. This provides a quasi-weight approach to these quantized integrals. In Connes' original approach, only finite cases (positive functionals) are considered.

\section{WEIGHTS AND QUASI-WEIGHTS ON A *-ALGEBRA}

Let $\mathcal{A}$ be a $*$-algebra, i.e. a linear associative involutive algebra. For a subspace $\mathcal{N}$ of $\mathcal{A}$, let $\mathcal{P}(\mathcal{N})=\left\{\sum_{k=1}^{n} x_{k}^{*} x_{k}: x_{k} \in \mathcal{N}(k=1,2, \cdots, n), n \in \mathbb{N}\right\}$, and call it the positive cone generated by $\mathcal{N}$.

Definition 2.1. (a) A map $\varphi: \mathcal{P}(\mathcal{A}) \rightarrow \mathbb{R}_{+} \cup\{\infty\}$ is said to be a weight on $\mathcal{P}(\mathcal{A})$ in $\mathcal{A}$ if

(i) $\varphi(x+y)=\varphi(x)+\varphi(y), \quad x, y \in \mathcal{P}(\mathcal{A})$,

(ii) $\varphi(\lambda x)=\lambda \varphi(x), \quad x \in \mathcal{P}(\mathcal{A}), \lambda \geq 0$.

(b) Let $\mathfrak{N}$ be a left ideal of $\mathcal{A}$. A map $\varphi: \mathcal{P}(\mathfrak{N}) \rightarrow \mathbb{R}_{+}$is called a quasi-weight on $\mathcal{P}(\mathfrak{N})$ in $\mathcal{A}$ if it satisfies the above (i) and (ii) for $\mathcal{P}(\mathfrak{N})$. In this case, we denote $\mathfrak{N}$ by $\mathfrak{N}_{\varphi}$.

Lemma 2.2. Let $\varphi$ be a weight on $\mathcal{P}(\mathcal{A})$ in $\mathcal{A}$. Let $\mathfrak{N}_{\varphi}=\left\{x \in \mathcal{A}: \varphi\left(x^{*} a^{*} a x\right)\right.$ $<\infty$ for all $a \in \mathcal{A}\}, \mathfrak{N}_{\varphi}^{0}=\left\{x \in \mathcal{A}: \varphi\left(x^{*} x\right)<\infty\right\}$. Then the following statements hold:

(i) $\mathfrak{N}_{\varphi}^{0}$ is a linear subspace of $\mathcal{A}$, and it need not be a left ideal.

(ii) $\mathfrak{N}_{\varphi}$ is a left ideal of $\mathcal{A}$, and $\varphi\left\lceil\mathcal{P}\left(\mathfrak{N}_{\varphi}\right)\right.$ is a quasi-weight (denoted by $\varphi_{q}$ and called a quasi-weight generated by $\varphi$ ).

(iii) If $\mathcal{A}$ has identity, then $\mathfrak{N}_{\varphi} \subset \mathfrak{N}_{\varphi}^{0}$.

(iv) If $\mathcal{A}$ is a $C^{*}$-algebra, then $\mathfrak{N}_{\varphi}^{0} \subset \mathfrak{N}_{\varphi}$.

We here state the basic theory of (unbounded) *-representations of $\mathcal{A}$. Let $\mathcal{D}$ be a dense subspace in a Hilbert space $\mathcal{H}$ and let $\mathcal{L}^{\dagger}(\mathcal{D})$ denote the set of all linear operators $X$ in $\mathcal{H}$ with the domain $\mathcal{D}$ for which $X \mathcal{D} \subset \mathcal{D}, \mathcal{D}\left(X^{*}\right) \supset \mathcal{D}$ and $X^{*} \mathcal{D} \subset \mathcal{D}$. Then $\mathcal{L}^{\dagger}(\mathcal{D})$ is a $*$-algebra under the usual operations and the involution $X \rightarrow X^{\dagger} \equiv X^{*}\left\lceil\mathcal{D}\right.$. A $*$-subalgebra of the $*$-algebra $\mathcal{L}^{\dagger}(\mathcal{D})$ is said to be an $O^{*}$-algebra on $\mathcal{D}$ in $\mathcal{H}$. A *-representation $\pi$ of $\mathcal{A}$ on a Hilbert space $\mathcal{H}$ with a domain $\mathcal{D}$ is a $*$-homomorphism of $\mathcal{A}$ into $\mathcal{L}^{\dagger}(\mathcal{D})$, and then we replace $\mathcal{D}$ and $\mathcal{H}$ by $\mathcal{D}(\pi)$ and $\mathcal{H}_{\pi}$, respectively. Let $\pi$ be a $*$-representation of $\mathcal{A}$. If $\mathcal{D}(\pi)$ is complete with the graph topology $t_{\pi}$ defined by the family of seminorms $\left\{\|\cdot\|_{\pi(x)} \equiv\|\cdot\|+\|\pi(x) \cdot\|: x \in \mathcal{A}\right\}$, then $\pi$ is said to be closed. It is well known that $\pi$ is closed if and only if $\mathcal{D}(\pi)=\bigcap_{x \in \mathcal{A}} \mathcal{D}(\overline{\pi(x)})$. The closure $\bar{\pi}$ of $\pi$ is defined 
by

$$
\mathcal{D}(\bar{\pi})=\bigcap_{x \in \mathcal{A}} \mathcal{D}(\overline{\pi(x)}) \text { and } \bar{\pi}(x) \xi=\overline{\pi(x)} \xi \text { for } x \in \mathcal{A}, \xi \in \mathcal{D}(\bar{\pi}) .
$$

Then $\bar{\pi}$ is the smallest closed extension of $\pi$. We put

$$
\mathcal{D}^{*}(\pi)=\bigcap_{x \in \mathcal{A}} \mathcal{D}\left(\pi(x)^{*}\right) \text { and } \pi^{*}(x) \xi=\pi\left(x^{*}\right)^{*} \xi \text { for } x \in \mathcal{A}, \xi \in \mathcal{D}^{*}(\pi) .
$$

Then $\pi^{*}$ is a representation of $\mathcal{A}$, but it is not necessarily a $*$-representation. If $\pi^{*}=\pi$, then $\pi$ is said to be self-adjoint. A weak commutant $\pi(\mathcal{A})_{\mathrm{w}}^{\prime}$ of $\pi$ is defined by

$$
\pi(\mathcal{A})_{\mathrm{w}}^{\prime}=\left\{C \in \mathcal{B}\left(\mathcal{H}_{\pi}\right):\langle C \pi(x) \xi, \eta\rangle=\left\langle C \xi, \pi\left(x^{*}\right) \eta\right\rangle \forall x \in \mathcal{A}, \quad \forall \xi, \eta \in \mathcal{D}(\pi)\right\},
$$

where $\mathcal{B}\left(\mathcal{H}_{\pi}\right)$ is the set of all bounded linear operators on $\mathcal{H}_{\pi}$, and it is a weakly closed $*$-invariant subspace of $\mathcal{B}\left(\mathcal{H}_{\pi}\right)$, but it is not necessarily an algebra. It is known that the self-adjointness of $\pi$ implies $\pi(\mathcal{A})_{\mathrm{w}}^{\prime} \mathcal{D}(\pi) \subset \mathcal{D}(\pi)$ and $\pi(\mathcal{A})_{\mathrm{w}}^{\prime} \mathcal{D}(\pi) \subset$ $\mathcal{D}(\pi)$ if and only if $\pi(\mathcal{A})_{\mathrm{w}}^{\prime}$ is a von Neumann algebra and $\overline{\pi(x)}$ is affiliated with the von Neumann algebra $\left(\pi(\mathcal{A})_{\mathrm{w}}^{\prime}\right)^{\prime}$ for each $x \in \mathcal{A}$. For more details we refer to [23], $[25]$.

Let $\varphi$ be a quasi-weight on $\mathcal{P}\left(\mathfrak{N}_{\varphi}\right)$ in $\mathcal{A}$. Let $\mathcal{D}(\varphi)$ be the subspace of $\mathcal{A}$ generated by $\left\{x^{*} x: x \in \mathfrak{N}_{\varphi}\right\}$. Since $\mathfrak{N}_{\varphi}$ is a left ideal, we have $\mathcal{D}(\varphi)=$ linear span of $\left\{y^{*} x: x, y \in \mathfrak{N}_{\varphi}\right\}$. Thus each $z=\sum_{k} \alpha_{k} y_{k}^{*} x_{k} \quad\left(\alpha_{k} \in \mathbb{C}, x_{k}, y_{k} \in \mathfrak{N}_{\varphi}\right)$ in $\mathcal{D}(\varphi)$ is represented as $z=\sum_{j} \beta_{j} z_{j}^{*} z_{j} \quad\left(\beta_{j} \in \mathbb{C}, z_{j} \in \mathfrak{N}_{\varphi}\right)$. Hence a linear functional on $\mathcal{D}(\varphi)$ into $\mathbb{C}$ is defined by $\varphi(z)=\sum_{j} \beta_{j} \varphi\left(z_{j}^{*} z_{j}\right)$. Then $\varphi$ satisfies the CauchySchwarz inequality : $\left|\varphi\left(y^{*} x\right)\right|^{2} \leq \varphi\left(y^{*} y\right) \varphi\left(x^{*} x\right) \quad\left(x, y \in \mathfrak{N}_{\varphi}\right)$. Now $N_{\varphi} \equiv\left\{x \in \mathfrak{N}_{\varphi}\right.$ : $\left.\varphi\left(x^{*} x\right)=0\right\}$ is a left ideal of $\mathfrak{N}_{\varphi}$. Let $\lambda_{\varphi}(x)=x+N_{\varphi}, x \in \mathfrak{N}_{\varphi}$. Then $X_{\varphi}=\mathfrak{N}_{\varphi} / N_{\varphi}$ is a pre-Hilbert space with inner product $\left\langle\lambda_{\varphi}(x), \lambda_{\varphi}(y)\right\rangle=\varphi\left(y^{*} x\right)$. Let $\mathcal{H}_{\varphi}$ be the Hilbert space obtained by the completion of $X_{\varphi}$. Let $\pi_{\varphi}^{0}$ be the representation of $\mathcal{A}$ on $\mathcal{H}_{\varphi}$ with domain $\mathcal{D}\left(\pi_{\varphi}^{0}\right)=X_{\varphi}$ as $\pi_{\varphi}^{0}(x) \lambda_{\varphi}(y)=\lambda_{\varphi}(x y)$. Let $\left(\pi_{\varphi}, \mathcal{D}\left(\pi_{\varphi}\right), \mathcal{H}_{\varphi}\right)$ be the closure of $\pi_{\varphi}^{0}$ [25]. Note that $\varphi\left(z^{*} x y\right)=\left\langle\pi_{\varphi}(x) \lambda_{\varphi}(y), \lambda_{\varphi}(z)\right\rangle \quad(x \in \mathcal{A}, y$ and $z$ in $\mathfrak{N}_{\varphi}$ ), and if $\mathcal{A}$ has identity, then $\mathcal{H}_{\varphi}=\left[\pi_{\varphi}(\mathcal{A}) \lambda_{\varphi}\left(\mathfrak{N}_{\varphi}\right)\right]^{-}$(norm closure), and $\mathcal{D}\left(\pi_{\varphi}\right)=\left[\pi_{\varphi}(\mathcal{A}) \lambda_{\varphi}\left(\mathfrak{N}_{\varphi}\right)\right]^{t_{\pi_{\varphi}(\mathcal{A})}}$ (closure in the graph topology $\left.t_{\pi_{\varphi}(\mathcal{A})}\right)$. The *-representation $\pi_{\varphi}$ is called the GNS-representation for $\varphi$. On the other hand, in Example 6.3 , we consider the quasi-weights defined by given $*$-representations of $\mathcal{A}$.

We shall need the concept of regular quasi-weights introduced in [18]. Let $\left\{f_{\alpha}\right\}$ be a net of positive linear functionals on $\mathcal{A}$. Define $\sup _{\alpha} f_{\alpha}: z \in \mathcal{P}(\mathcal{A}) \rightarrow \sup _{\alpha} f_{\alpha}(z) \in$ $[0, \infty]$. Let $\mathfrak{N}_{\sup _{\alpha} f_{\alpha}}=\left\{x \in \mathcal{A}: \sup _{\alpha} f_{\alpha}\left(x^{*} x\right)<\infty\right\}$ a subspace of $\mathcal{A}$. It is shown in

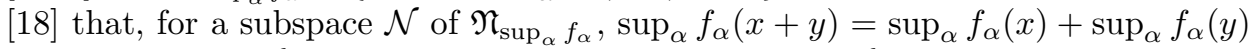
for all $x, y$ in $\mathcal{P}(\mathcal{N})$ iff $\left\{f_{\alpha}\right\}$ has the net property for $\mathcal{P}(\mathcal{N})$ in the sense that, given a finite set $\left\{x_{1}, x_{2}, \cdots, x_{m}\right\}$ in $\mathcal{N}$, there exists a subsequence $\left\{\alpha_{n}\right\}$ of $\{\alpha\}$ such that $\lim _{n \rightarrow \infty} f_{\alpha_{n}}\left(x_{k}^{*} x_{k}\right)=\sup _{\alpha} f_{\alpha}\left(x_{k}^{*} x_{k}\right), k=1,2, \cdots, m$. In this case, the restriction of the map $\sup _{\alpha} f_{\alpha}$ to $\mathcal{P}(\mathcal{N})$ is denoted by $\operatorname{Sup}_{\alpha} f_{\alpha}\left\lceil\mathcal{P}(N)\right.$. If $\left\{f_{\alpha}\right\}$ has the net property for $\mathcal{P}\left(\mathfrak{N}_{\sup _{\alpha} f_{\alpha}}^{0}\right)$, then the restriction of $\sup _{\alpha} f_{\alpha}$ to $\mathcal{P}\left(\mathfrak{N}_{\sup _{\alpha} f_{\alpha}}\right)$ is denoted by $\operatorname{Sup}_{\alpha} f_{\alpha}$.

Definition 2.3. Let $\varphi$ and $\psi$ be quasi-weights in $\mathcal{A}$. We write $\psi \leq \varphi$ if $\mathfrak{N}_{\varphi} \subset \mathfrak{N}_{\psi}$ and $\psi\left(x^{*} x\right) \leq \varphi\left(x^{*} x\right)$ for all $x \in \mathfrak{N}_{\varphi}$. We write $\psi \prec \varphi$ if $\mathfrak{N}_{\varphi}=\mathfrak{N}_{\psi}$ and $\psi\left(x^{*} x\right) \leq$ 
$\varphi\left(x^{*} x\right)$ for all $x \in \mathfrak{N}_{\varphi}$. A positive linear functional $f$ on $\mathcal{A}$ is said to be $\varphi$-majorized if $f \leq \varphi$ and $|f(x)|^{2} \leq \varphi\left(x^{*} x\right)$ for all $x \in \mathfrak{N}_{\varphi}$.

We have the following lemma.

Lemma 2.4. Let $\varphi$ and $\psi$ be quasi-weights in $\mathcal{A}$. Suppose $\psi \leq \varphi$. Then there exists a continuous linear map $K_{\varphi, \psi}: \mathcal{H}_{\varphi} \rightarrow \mathcal{H}_{\psi}$ defined by $K_{\varphi, \psi} \lambda_{\varphi}(x)=\lambda_{\psi}(x)$ $\left(x \in \mathfrak{N}_{\varphi}\right)$. Further, $K_{\varphi, \psi}$ satisfies the following:

(i) $K_{\varphi, \psi}^{*} K_{\varphi, \psi} \in \pi_{\varphi}(\mathcal{A})_{\mathrm{w}}^{\prime}$,

(ii) $\psi\left(x^{*} x\right)=\left\langle K_{\varphi, \psi}^{*} K_{\varphi, \psi} \lambda_{\varphi}(x), \lambda_{\varphi}(x)\right\rangle$, for each $x \in \mathfrak{N}_{\varphi}$.

Definition 2.5. A quasi-weight $\varphi$ on $\mathcal{P}(\mathcal{A})$ is called regular if $\varphi=\sup _{\alpha} f_{\alpha}\left\lceil\mathcal{P}\left(\mathfrak{N}_{\varphi}\right)\right.$ for some net $\left\{f_{\alpha}\right\}$ of $\varphi$-majorized positive linear functionals on $\mathcal{A}$. A weight $\varphi$ is regular if $\varphi=\sup _{\alpha} f_{\alpha}$ for some net $\left\{f_{\varphi}\right\}$ of $\varphi$-majorized positive linear functionals on $\mathcal{A}$.

The proof of the following lemma is similar to the proof of [18, Theorem 3.5].

Lemma 2.6. Let $\varphi$ be a regular quasi-weight in $\mathcal{A}$. Then there exist a net $\left\{C_{\beta}\right\}$ in $\pi_{\varphi}(\mathcal{A})_{\mathrm{w}}^{\prime}, 0 \leq C_{\beta} \leq I$, and a net $\left\{\xi_{\beta}\right\}$ in $\mathcal{D}\left(\pi_{\varphi}{ }^{*}\right)$ such that

(i) $C_{\beta} \lambda_{\varphi}(x)=\pi_{\varphi}{ }^{*}(x) \xi_{\beta}$, for each $x \in \mathfrak{N}_{\varphi}$,

(ii) $C_{\beta} \rightarrow I$ strongly.

We need the following lemma in Section 5.

Lemma 2.7. Let $\varphi$ be a regular quasi-weight in $\mathcal{A}$ such that $\pi_{\varphi}(\mathcal{A})_{\mathrm{w}}^{\prime}$ is a von Neumann algebra. For each $C \in \pi_{\varphi}(\mathcal{A})_{\mathrm{w}}^{\prime}, C \geq 0$, we put

$$
\varphi_{C}\left(x^{*} x\right)=\left\langle C \lambda_{\varphi}(x), \lambda_{\varphi}(x)\right\rangle, x \in \mathfrak{N}_{\varphi} .
$$

Then $\varphi_{C}$ is a quasi-weight on $\mathcal{P}(\mathcal{A})$.

Proof. By Lemma 2.6, there exist a net $\left\{C_{\beta}\right\}$ in $\pi_{\varphi}(\mathcal{A})_{\mathrm{w}}^{\prime}$ and a net $\left\{\xi_{\beta}\right\}$ in $\mathcal{D}\left(\pi_{\varphi}{ }^{*}\right)$ satisfying the conditions in Lemma 2.6. For $x \in \mathfrak{N}_{\varphi}$ we have

$$
\begin{aligned}
\varphi_{C}\left(x^{*} x\right) & =\left\langle C \lambda_{\varphi}(x), \lambda_{\varphi}(x)\right\rangle \\
& =\lim _{\beta}\left\langle C \lambda_{\varphi}(x), C_{\beta} \lambda_{\varphi}(x)\right\rangle \\
& =\lim _{\beta}\left\langle C \lambda_{\varphi}(x), \pi_{\varphi}{ }^{*}(x) \xi_{\beta}\right\rangle \\
& =\lim _{\beta}\left\langle C \lambda_{\varphi}(x), \widehat{\pi}_{\varphi}^{*}(x) \xi_{\beta}\right\rangle \\
& =\lim _{\beta}\left\langle\widehat{\pi_{\varphi}}\left(x^{*}\right) C \lambda_{\varphi}(x), \xi_{\beta}\right\rangle \\
& =\lim _{\beta}\left\langle C \widehat{\pi_{\varphi}}\left(x^{*}\right) \lambda_{\varphi}(x), \xi_{\beta}\right\rangle \\
& =\lim _{\beta}\left\langle C \pi_{\varphi}\left(x^{*}\right) \lambda_{\varphi}(x), \xi_{\beta}\right\rangle \\
& =\lim _{\beta}\left\langle C \lambda_{\varphi}\left(x^{*} x\right), \xi_{\beta}\right\rangle,
\end{aligned}
$$

where $\widehat{\pi_{\varphi}}$ is the induced extension of $\pi_{\varphi}\left[25\right.$, Section 8.5]. This implies that $\varphi_{C}$ is a quasi-weight on $\mathcal{P}(\mathcal{A})$.

\section{BOUNDEDNESS AND ADMISSIBILITY}

Let $\varphi$ be a quasi-weight on $\mathcal{P}\left(\mathfrak{N}_{\varphi}\right)$ in a $*$-algebra $\mathcal{A}$. We define the $\varphi$-bounded part $\mathcal{A}_{\varphi}^{b}$ of $\mathcal{A}$ by

$$
\mathcal{A}_{\varphi}^{b}=\left\{a \in \mathcal{A}: \exists k_{a}>0 \text { such that } \varphi\left(x^{*} a^{*} a x\right) \leq k_{a} \varphi\left(x^{*} x\right), \quad \forall x \in \mathfrak{N}_{\varphi}\right\} .
$$


For $a \in \mathcal{A}_{\varphi}^{b}$ we put

$$
p_{\varphi}(a)=\sup \left\{\frac{\varphi\left(x^{*} a^{*} a x\right)^{\frac{1}{2}}}{\varphi\left(x^{*} x\right)^{\frac{1}{2}}}: x \in \mathfrak{N}_{\varphi}, \quad \varphi\left(x^{*} x\right) \neq 0\right\} .
$$

We have the following:

Lemma 3.1. (1) $\mathcal{A}_{\varphi}^{b}=\left\{a \in \mathcal{A}: \overline{\pi_{\varphi}(a)} \in \mathcal{B}\left(\mathcal{H}_{\varphi}\right)\right\}$, and $\mathcal{A}_{\varphi}^{b}$ is a $*$-subalgebra of $\mathcal{A}$.

(2) $p_{\varphi}(a)=\left\|\overline{\pi_{\varphi}(a)}\right\|, a \in \mathcal{A}_{\varphi}^{b}$, and $p_{\varphi}$ is a $C^{*}$-seminorm on $\mathcal{A}_{\varphi}^{b}$.

Definition 3.2. A quasi-weight $\varphi$ on $\mathcal{P}\left(\mathfrak{N}_{\varphi}\right)$ in a $*$-algebra $\mathcal{A}$ is admissible if $\mathcal{A}=\mathcal{A}_{\varphi}^{b}$.

Let $\varphi$ be a weight on $\mathcal{P}(\mathcal{A})$ in a $*$-algebra $\mathcal{A}$. We put

$$
\mathcal{A}_{\varphi}^{b s}=\left\{a \in \mathcal{A}: \exists k_{a}>0 \text { such that } \varphi\left(x^{*} a^{*} a x\right) \leq k_{a} \varphi\left(x^{*} x\right), \forall x \in \mathcal{A}\right\} .
$$

Then $\mathcal{A}_{\varphi}^{b s}$ is a subalgebra of $\mathcal{A}$ contained in $\mathcal{A}_{\varphi_{q}}^{b}$.

We do not know whether $\mathcal{A}_{\varphi}^{b s}$ is $*$-invariant or not.

Definition 3.3. A weight $\varphi$ on $\mathcal{P}(\mathcal{A})$ is said to be admissible if the quasi-weight $\varphi_{q}=\varphi\left\lceil\mathcal{P}\left(\mathfrak{N}_{\varphi}\right)\right.$ defined by $\varphi$ is admissible, i.e. $\mathcal{A}=\mathcal{A}_{\varphi_{q}}^{b}$. Further, $\varphi$ is said to be strongly admissible if $\mathcal{A}=\mathcal{A}_{\varphi}^{b s}$.

Lemma 3.4. Let $\varphi$ be a weight on $\mathcal{P}(\mathcal{A})$. Then the following are equivalent.

(i) $\varphi$ is strongly admissible.

(ii) $\varphi$ is admissilbe and $\mathfrak{N}_{\varphi}^{0} \subset \mathfrak{N}_{\varphi}$.

Proposition 3.5. Let $\varphi$ be a quasi-weight on $\mathcal{P}\left(\mathfrak{N}_{\varphi}\right)$ in a *-algebra $\mathcal{A}$.

(1) The following are equivalent.

(i) $\varphi$ is admissible.

(ii) There exist a submultiplicative seminorm $p$ on $\mathcal{A}$ and a scalar $\gamma>0$ such that $\left|\varphi\left(x^{*} a x\right)\right| \leq \gamma p(a) \varphi\left(x^{*} x\right)$ for each $x \in \mathfrak{N}_{\varphi}$ and $a \in \mathcal{A}$.

(2) $\varphi$ is admissible if there exist a submultiplicative seminorm $p$ on $\mathcal{A}$ and a scalar $\gamma>0$ such that $\left|\varphi\left(x^{*} a x\right)\right| \leq \gamma p\left(x^{*} a x\right)$ for each $a \in \mathcal{A}$ and $x \in \mathfrak{N}_{\varphi}$.

We do not know whether the converse of (2) holds or not. For the proof of (2), we shall use the following [7, Proposition 2.8]:

Lemma 3.6. Let $q$ be any submultiplicative seminorm on an algebra $\mathcal{A}$. Then $\lim _{n \rightarrow \infty} q\left(x^{n}\right)^{\frac{1}{n}}$ exists, and $\lim _{n \rightarrow \infty} q\left(x^{n}\right)^{\frac{1}{n}}=\inf _{n} q\left(x^{n}\right)^{\frac{1}{n}}$.

Proof of Proposition 3.5. (1) For $a \in \mathcal{A}, x \in \mathfrak{N}_{\varphi}$, we have $a x \in \mathfrak{N}_{\varphi}$ and $x^{*} a x \in$ $\mathcal{D}(\varphi)$. Thus statement (ii) makes sense. Obviously (ii) implies (i). Suppose (i) holds; then by the Cauchy-Schwartz inequality for $\varphi$ in $\mathcal{D}(\varphi)$ we have

$$
\left|\varphi\left(x^{*} a x\right)\right|^{2} \leq \varphi\left((a x)^{*} a x\right) \varphi\left(x^{*} x\right) \leq p_{\varphi}(a)^{2} \varphi\left(x^{*} x\right)^{2} \quad\left(x \in \mathfrak{N}_{\varphi}, a \in \mathcal{A}\right),
$$

which implies (ii).

(2) Define $q(x)=\max \left(p(x), p\left(x^{*}\right)\right), x \in \mathcal{A}$. Then $q$ is a $*$-invariant submultiplicative seminorm on $\mathcal{A}$, and $\left|\varphi\left(x^{*} a x\right)\right| \leq \gamma q\left(x^{*} a x\right)$ holds for all $x \in \mathfrak{N}_{\varphi}, a \in \mathcal{A}$. 
Then we have

$$
\begin{aligned}
\varphi\left(x^{*} a^{*} a x\right) & =\varphi\left(x^{*}\left(a^{*} a x\right)\right) \\
& =\left\langle\lambda_{\varphi}\left(a^{*} a x\right), \lambda_{\varphi}(x)\right\rangle \\
& \leq\left\|\lambda_{\varphi}\left(a^{*} a x\right)\right\|\left\|\lambda_{\varphi}(x)\right\| \\
& =\left\langle\lambda_{\varphi}\left(a^{*} a x\right), \lambda_{\varphi}\left(a^{*} a x\right)\right\rangle^{\frac{1}{2}}\left\langle\lambda_{\varphi}(x), \lambda_{\varphi}(x)\right\rangle^{\frac{1}{2}} \\
& =\varphi\left(x^{*}\left(a^{*} a\right)^{2} x\right)^{\frac{1}{2}} \varphi\left(x^{*} x\right)^{\frac{1}{2}} .
\end{aligned}
$$

By induction, for any $n \in \mathbb{N}$ we have

$$
\begin{aligned}
\varphi\left(x^{*}\left(a^{*} a\right) x\right)^{2^{n+1}} & \leq \varphi\left(x^{*}\left(a^{*} a\right)^{2^{n+1}} x\right) \varphi\left(x^{*} x\right)^{2^{n+1}-1} \\
& \leq \gamma q\left(x^{*}\left(a^{*} a\right)^{2^{n+1}} x\right) \varphi\left(x^{*} x\right)^{2^{n+1}-1} \\
& \leq \gamma q\left(\left(a^{*} a\right)^{2^{n+1}}\right) q(x)^{2} \varphi\left(x^{*} x\right)^{2^{n+1}-1} .
\end{aligned}
$$

Therefore we have

$$
\varphi\left(x^{*} a^{*} a x\right) \leq \gamma^{\frac{1}{2^{n+1}}} q\left(\left(a^{*} a\right)^{2^{n+1}}\right)^{\frac{1}{2^{n+1}}} q(x)^{\frac{1}{2^{n}}} \varphi\left(x^{*} x\right)^{1-\frac{1}{2^{n+1}}} .
$$

Letting $n \rightarrow \infty$, we get $\varphi\left(x^{*} a^{*} a x\right) \leq r_{q}\left(a^{*} a\right) \varphi\left(x^{*} x\right)\left(x \in \mathfrak{N}_{\varphi}, a \in \mathcal{A}\right)$, where $r_{q}\left(a^{*} a\right)=\lim _{n \rightarrow \infty} q\left(\left(a^{*} a\right)^{n}\right)^{\frac{1}{n}}<\infty$, which implies that $\varphi$ is admissible.

Next we consider when every quasi-weight on a $*$-algebra $\mathcal{A}$ is admissible. The notion of quasi-weights imposes a certain boundedness structure on $\mathcal{A}$. Let $W_{q}(\mathcal{A})$ denote the set of all quasi-weights on $\mathcal{A}$. An element $a \in \mathcal{A}$ is said to be quasi-weight bounded if for each $\varphi \in W_{q}(\mathcal{A})$, there is a scalar $k_{a, \varphi}>0$ such that $\varphi\left(x^{*} a^{*} a x\right) \leq$ $k_{a, \varphi} \varphi\left(x^{*} x\right)$ for all $x \in \mathfrak{N}_{\varphi}$. Further, $a$ is uniformly quasi-weight bounded if $k_{a} \equiv$ $\sup \left\{k_{a, \varphi}: \varphi \in W_{q}(\mathcal{A})\right\}<\infty$. Let $\mathcal{A}^{b}\left(\right.$ resp. $\mathcal{A}^{u b}$ ) denote the set of all quasi-weight bounded (resp. uniformly quasi-weight bounded) elements of $\mathcal{A}$.

Proposition 3.7. (1) $\mathcal{A}^{b}=\bigcap\left\{\mathcal{A}_{\varphi}^{b}: \varphi \in W_{q}(\mathcal{A})\right\}$, and it is a $*$-subalgebra of $\mathcal{A}$ containing $\mathcal{A}^{u b}$ as a *-subalgebra.

(2) Let $\tau$ denote the (not necessarily Hausdorff) topology on $\mathcal{A}^{b}$ defined by the $C^{*}$ seminorms $\left\{p_{\varphi}: \varphi \in W_{q}(\mathcal{A})\right\}$. For $a \in \mathcal{A}^{u b}$, let $|a|_{\infty}=\sup \left\{p_{\varphi}(a): \varphi \in W_{q}(\mathcal{A})\right\}$. Then $|\cdot|_{\infty}$ is a $C^{*}$-seminorm on $\mathcal{A}^{u b}$, and $\left(\mathcal{A}^{u b},|\cdot|_{\infty}\right) \hookrightarrow\left(\mathcal{A}^{b}, \tau\right)$ is continuous.

(3) The following are equivalent.

(a) $|\cdot|_{\infty}$ is a norm on $\mathcal{A}^{u b}$.

(b) $\tau$ is Hausdorff on $\mathcal{A}^{b}$.

(c) There exists $\left\{\varphi_{\lambda}: \lambda \in \Lambda\right\} \subset W_{q}(\mathcal{A})$ which is separating in the sense that for any $a \in \mathcal{A}, \varphi_{\lambda}\left(x^{*} a^{*} a x\right)=0\left(\forall x \in \mathfrak{N}_{\varphi_{\lambda}}, \forall \lambda \in \Lambda\right)$ implies $a=0$.

(4) Every quasi-weight in $\mathcal{A}$ is admissible iff $\mathcal{A}=\mathcal{A}^{b}$.

We omit the easy proof. In the following, we illustrate the above structures, and show that the notion of Allan boundedness [1] is included in it.

Proposition 3.8. Let $(\mathcal{A},\|\cdot\|)$ be a Banach $*$-algebra. Then each quasi-weight in $\mathcal{A}$ is admissible, $\mathcal{A}=\mathcal{A}^{u b}$, and $|\cdot|_{\infty}=p_{\infty}(\cdot)$ (the Gelfand-Naimark pseudonorm on $\mathcal{A})$. Further, if $\mathcal{A}$ is a $C^{*}$-algebra, then $\|\cdot\|=|\cdot|_{\infty}$.

Proof. For a quasi-weight $\varphi$ on $\mathcal{P}\left(\mathfrak{N}_{\varphi}\right)$ in $\mathcal{A}$, the $*$-representation $\pi_{\varphi}: \mathcal{A} \rightarrow$ $\mathcal{L}^{\dagger}\left(\mathcal{D}\left(\pi_{\varphi}\right)\right)$ can be extended to the unitization $\mathcal{A}_{1}$ of $\mathcal{A}$. Hence we assume that $\mathcal{A}$ has identity. Then for any $\xi \in \mathcal{D}\left(\pi_{\varphi}\right)$, the positive linear functional $\omega_{\xi}(a)=$ $\left\langle\pi_{\varphi}(a) \xi, \xi\right\rangle(a \in \mathcal{A})$ is continuous [7, Corollary 37.9], and $\left|\omega_{\xi}(a)\right| \leq\left\|\omega_{\xi}\right\|\|a\|=$ $\omega_{\xi}(1)\|a\|, \quad \forall a \in \mathcal{A}$. Hence $\left\|\pi_{\varphi}(a) \xi\right\| \leq\|a\|\|\xi\| \quad\left(a \in \mathcal{A}, \xi \in \mathcal{D}\left(\pi_{\varphi}\right)\right)$. Thus $\varphi$ is admissible, and $p_{\varphi}(a) \leq\|a\|$. Since the Gelfand-Naimark pseudonorm is the 
greatest $\mathrm{C}^{*}$-seminorm on $\mathcal{A}$, we have $p_{\varphi}(a) \leq p_{\infty}(a)(a \in \mathcal{A})$. Thus $\mathcal{A}=\mathcal{A}^{u b}$ and $|a|_{\infty} \leq p_{\infty}(a)$. On the other hand, since every positive linear functional $f$ on $\mathcal{A}$ defines a finite weight $\varphi=f\left\lceil_{\mathcal{P}(A)}\right.$, it follows from [7, Corollary 39.12] that

$$
\begin{aligned}
p_{\infty}(a)^{2} & =\sup _{f}\left\{\sup \left\{\frac{f\left(x^{*} a^{*} a x\right)}{f\left(x^{*} x\right)}: x \in \mathcal{A}, f\left(x^{*} x\right) \neq 0\right\}\right\} \\
& \leq \sup \left\{p_{\varphi}(a)^{2}: \varphi \in W_{q}(\mathcal{A})\right\} \\
& =|a|_{\infty}^{2} . \quad \square
\end{aligned}
$$

Following Palmer, a *-algebra $\mathcal{A}$ is a $B G^{*}$-algebra if every *-representation $(\pi, \mathcal{D}(\pi), \mathcal{H})$ of $\mathcal{A}$ necessarily maps $\mathcal{A}$ into bounded operators [5]. Then $\sigma=\sum_{\pi}^{\oplus} \pi$ is also a bounded operator representation, and $q_{\infty}(a)=\|\sigma(a)\|(a \in \mathcal{A})$ defines the greatest $\mathrm{C}^{*}$-seminorm on $\mathcal{A}$. A $U^{*}$-algebra is a $*$-algebra $\mathcal{A}$ which is a linear span of its quasi-unitary elements. A Banach $*$-algebra is a $\mathrm{U}^{*}$-algebra, and a $\mathrm{U}^{*}$-algebra is a $\mathrm{BG}^{*}$-algebra. The following proposition can be proved in the same way as the previous one.

Proposition 3.9. Let $\mathcal{A}$ be a $B G^{*}$-algebra. Then $\mathcal{A}=\mathcal{A}^{u b}$ and $|\cdot|_{\infty}=p_{\infty}(\cdot)$. In particular, every quasi-weight in $\mathcal{A}$ is admissible.

Now let $\mathcal{A}$ be a locally convex $*$-algebra. An element $a \in \mathcal{A}$ is called (Allan) bounded [1] if there exists a $\lambda>0$ such that $\left\{\left(\lambda^{-1} a\right)^{n}: n \in \mathbb{N}\right\}$ is bounded. Let $\mathcal{A}_{0}$ be the bounded part of $\mathcal{A}$, consisting of all Allan bounded elements of $\mathcal{A}$. We denote by $\mathcal{A}\left[\mathcal{A}_{0}^{h}\right]$ the $*$-subalgebra of $\mathcal{A}$ generated by $\left(\mathcal{A}_{0}\right)_{h}=\left\{h \in \mathcal{A}: h=h^{*}\right\}$. Since $\mathcal{A}_{0}$ is not even a subspace in general, $\mathcal{A}\left[\mathcal{A}_{0}^{h}\right] \not \subset \mathcal{A}_{0}$. If $\mathcal{A}_{0}$ is a $*$-sublagebra of $\mathcal{A}$ (in particular, if $\mathcal{A}$ is commutative), then $\mathcal{A}_{0}=\mathcal{A}\left[\mathcal{A}_{0}^{h}\right]$. By $\mathcal{B}$ we denote the collection of all subsets $\mathbf{B}$ of $\mathcal{A}$ such that $\mathbf{B}$ is bounded, closed and absolutely convex, and $\mathbf{B}^{2} \subset \mathbf{B}$. For each $\mathbf{B} \in \mathcal{B}$ we put

$$
\begin{aligned}
& \mathcal{A}(\mathbf{B})=\{\lambda x: \lambda \in \mathbb{C}, x \in \mathbf{B}\}, \\
& \|x\|_{\mathbf{B}}=\inf \{\lambda>0: x \in \lambda \mathbf{B}\}, \quad x \in \mathcal{A}(\mathbf{B}) .
\end{aligned}
$$

Then $\mathcal{A}(\mathbf{B})$ is a normed algebra with the norm $\|\cdot\|_{\mathbf{B}}$. A locally convex algebra $\mathcal{A}$ is said to be pseudo-complete if $\left(\mathcal{A}(\mathbf{B}),\|\cdot\|_{\mathbf{B}}\right)$ is complete for every $\mathbf{B} \in \mathcal{B}$. If $\mathcal{A}$ is sequentially complete, then it is pseudo-complete, but the converse doesn't hold in general [1, Example 1]. The radius of boundedness $\beta(x)$ of $x \in \mathcal{A}$ is defined [1] by

$$
\beta(x)=\inf \left\{\lambda>0:\left\{\left(\lambda^{-1} x\right)^{n}: n \in \mathbb{N}\right\} \text { is bounded }\right\},
$$

where inf $\varnothing=\infty$.

Lemma 3.10. Let $\mathcal{A}$ be a pseudo-complete locally convex $*$-algebra. Then $\mathcal{A}\left[\mathcal{A}_{0}^{h}\right] \subset$ $\mathcal{A}^{u b}$, and for all $a \in\left(\mathcal{A}_{0}\right)_{h},|a|_{\infty} \leq \beta(a)$ (the radius of boundedness of a). Further, if $\varphi$ is a weight on $\mathcal{P}(\mathcal{A})$, then $\mathcal{A}\left[\mathcal{A}_{0}^{h}\right] \subset \mathcal{A}_{\varphi}^{b s} \cap\left(\mathcal{A}_{\varphi}^{b s}\right)^{*}$.

Proof. We first show the following

Statement. Let $\varepsilon>0$. Let $a=a^{*}$. Then there exists $b=b^{*}$ in $\mathcal{A}$ such that

$$
x^{*} a^{2} x+\left(\beta(a)^{2}+\varepsilon\right)(x-b x)^{*}(x-b x)=\left(\beta(a)^{2}+\varepsilon\right) x^{*} x
$$

for all $x \in \mathcal{A}$. 
This is proved by a square root lemma argument. Let $x_{0}=\frac{1}{\left(\beta(a)^{2}+\varepsilon\right)} a^{2}$. Then $\beta\left(x_{0}\right)<1$. Hence by [1, Corollary 2.17], there exists an element $\mathbf{B}$ in $\mathcal{B}$ such that $x_{0} \in \mathcal{A}(\mathbf{B})$ and $\left\|x_{0}\right\|_{\mathbf{B}}<1$. The scalar series $f(z)=-\sum_{n=1}^{\infty}\left(\begin{array}{l}\frac{1}{2} \\ n\end{array}\right)(-z)^{n}$ converges for all $z,|z| \leq 1$, and $2 f(z)-f(z)^{2}=z$. Since $\left(\mathcal{A}(\mathbf{B}),\|\cdot\|_{\mathbf{B}}\right)$ is a Banach algebra, this implies that $\sum_{n=1}^{\infty}\left(\begin{array}{c}\frac{1}{2} \\ n\end{array}\right)\left(-x_{0}\right)^{n}$ converges in $\|\cdot\|_{\mathbf{B}}$ to an element $b \in \mathcal{A}(\mathbf{B}) \subset \mathcal{A}_{0}$ such that $2 b-b^{2}=x_{0}=\frac{1}{\beta(a)^{2}+\varepsilon} a^{2}$. Since $x_{0}=x_{0}^{*}$, we have $b=b^{*}$. Thus, $x^{*} a^{2} x=\left(\beta(a)^{2}+\varepsilon\right)\left(2 x^{*} b x-x^{*} b^{2} x\right)$. This immediately gives

$$
x^{*} a^{2} x+\left(\beta(a)^{2}+\varepsilon\right)(x-b x)^{*}(x-b x)=\left(\beta(a)^{2}+\varepsilon\right) x^{*} x .
$$

This implies that $\varphi\left(x^{*} a^{2} x\right) \leq\left(\beta(a)^{2}+\varepsilon\right) \varphi\left(x^{*} x\right)$, for all $\varphi \in W_{q}(\mathcal{A})$. Letting $\varepsilon \rightarrow 0$, we get $\varphi\left(x^{*} a^{2} x\right) \leq \beta(a)^{2} \varphi\left(x^{*} x\right)$; hence $p_{\varphi}(a)^{2}=p_{\varphi}\left(a^{2}\right) \leq \beta(a)^{2}$. Thus, $a \in \mathcal{A}^{u b}$ with $|a|_{\infty} \leq \beta(a)$. It follows that $\mathcal{A}\left[\mathcal{A}_{0}^{h}\right] \subset \mathcal{A}^{u b}$. The assertion about weight can be proved similarly.

Lemma 3.11. Let $\varphi$ be a quasi-weight on $\mathcal{P}\left(\mathfrak{N}_{\varphi}\right)$ in a locally convex *-algebra $\mathcal{A}$. Assume that $\varphi$ is continuous on $\mathcal{D}(\varphi)$. Then $\mathcal{A}\left[\mathcal{A}_{0}^{h}\right] \subset \mathcal{A}_{\varphi}^{b}$.

Proof. For each $x \in \mathfrak{N}_{\varphi}$ we define a continuous positive linear functional $\varphi_{x}$ on $\mathcal{A}$ by $\varphi_{x}(a)=\varphi\left(x^{*} a x\right), a \in \mathcal{A}$. By arguments as in Proposition 3.5 (2) we have

$$
\left|\varphi_{x}(h)\right|^{2^{n}} \leq \varphi\left(x^{*} x\right)^{2^{n}-1} \varphi_{x}\left(h^{2^{n}}\right)
$$

for all $h=h^{*}$ in $\mathcal{A}$ and all $n \in \mathbb{N}$. Hence it follows from [1, Proposition 2.18] that $\varphi\left(x^{*} h^{2} x\right) \leq \varphi\left(x^{*} x\right) \beta(h)^{2}$, which implies $h \in \mathcal{A}_{\varphi}^{b}$.

Lemmas 3.10 and 3.11 give the following.

Theorem 3.12. Let $\mathcal{A}$ be a locally convex $*$-algebra with $\mathcal{A}=\mathcal{A}_{0}$.

(1) If $\mathcal{A}$ is pseudo-complete, then every quasi-weight and every weight is admissible, $\mathcal{A}=\mathcal{A}^{u b}$, and $|a|_{\infty} \leq \beta\left(a^{*} a\right)^{\frac{1}{2}}$ for all $a \in \mathcal{A}$.

(2) If $\varphi$ is a quasi-weight on $\mathcal{P}\left(\mathfrak{N}_{\varphi}\right)$ in $\mathcal{A}$ such that $\varphi$ is continuous on $\mathcal{D}(\varphi)$, then $\varphi$ is admissible.

Corollary 3.13. Let $\mathcal{A}$ be a locally convex *-algebra with $\mathcal{A}=\mathcal{A}_{0}$. Let $\pi$ be a nondegenerate *-representation of $\mathcal{A}$. If $\mathcal{A}$ is pseudo-complete or if $\pi$ is weakly continuous, then $\pi$ is a bounded operator representation.

Proof. For $\xi \in \mathcal{D}(\pi)$, let $f_{\xi}(a)=\left(\omega_{\xi} \circ \pi\right)(a)=\langle\pi(a) \xi, \xi\rangle, \quad a \in \mathcal{A}$. Let $\mathcal{A}$ be pseudo-complete. By Lemma 3.10 we have $\|\pi(a) \pi(x) \xi\|^{2} \leq \beta(a)^{2}\|\pi(x) \xi\|^{2}$ for all $a=a^{*} \in \mathcal{A}$ and all $x \in \mathcal{A}$. Since $\pi$ is non-degenerate, it follows that $\pi(a)$ is bounded for all $a \in \mathcal{A}$. Now assume that $\pi$ is weakly continuous. Then, since each $f_{\xi}$ is continuous, it follows from Lemma 3.11 that $\|\pi(a) \pi(x) \xi\|^{2}=f_{\xi}\left(x^{*} a^{2} x\right) \leq$ $\beta(a)^{2} f_{\xi}\left(x^{*} x\right)=\beta(a)^{2}\|\pi(x) \xi\|^{2}$ for $a=a^{*} \in \mathcal{A}$ and $x \in \mathcal{A}$, which implies that $\pi$ maps $\mathcal{A}$ into bounded operators.

Given a locally convex *-algebra $\mathcal{A}$, define $\mathcal{B}^{*}=\left\{\mathbf{B} \in \mathcal{B} ; \mathbf{B}^{*}=\mathbf{B}\right\}$. Then $\mathcal{A}$ is said to be a $G B^{*}$-algebra [2], [12] if the following hold:

(i) $\mathcal{A}$ is symmetric.

(ii) The collection $\mathcal{B}^{*}$ admits the greatest member $\mathbf{B}_{0}$. 
(iii) The $*$-subalgebra $\mathcal{A}\left(\mathbf{B}_{0}\right)=\left\{\lambda x: \lambda \in \mathbb{C}, x \in \mathbf{B}_{0}\right\}$ is a $\mathrm{C}^{*}$-algebra with norm $\|x\|_{\mathbf{B}_{0}}=\inf \left\{\lambda>0: \lambda^{-1} x \in \mathbf{B}_{0}\right\}$.

A particular case of $\mathrm{GB}^{*}$-algebras is a projective limit of $\mathrm{C}^{*}$-algebras called pro- $\mathrm{C}^{*}$ algebras [21], i.e. a complete locally convex $*$-algebra whose topology is determined by the family $\mathrm{s}(\mathcal{A})$ of all continuous $\mathrm{C}^{*}$-seminorms. In this case,

$$
\mathbf{B}_{0}=\left\{a \in \mathcal{A}: \sup _{p \in \mathrm{s}(\mathcal{A})} p(a) \leq 1\right\} \quad \text { and } \quad\|a\|_{\mathbf{B}_{0}}=\sup _{p \in \mathrm{s}(\mathcal{A})} p(a) .
$$

Proposition 3.14. Let $\mathcal{A}$ be a pseudo-complete locally convex $G B^{*}$-algebra. Then $\mathcal{A}^{u b}=\mathcal{A}\left(\mathbf{B}_{0}\right)$ and $|\cdot|_{\infty}=\|\cdot\|_{\mathbf{B}_{0}}$.

Proof. By [12], $\mathcal{A}\left(\mathbf{B}_{0}\right)_{h} \subset\left(\mathcal{A}_{0}\right)_{h}$. Hence by Lemma 3.10, $\mathcal{A}\left(\mathbf{B}_{0}\right) \subset \mathcal{A}\left[\mathcal{A}_{0}^{h}\right] \subset \mathcal{A}^{u b}$, and by a standard $\mathrm{C}^{*}$-algebra argument, $|a|_{\infty}=\|a\|_{\mathbf{B}_{0}}$ for all $a \in \mathcal{A}\left(\mathbf{B}_{0}\right)$. Now let $a \in \mathcal{A}^{u b}$. Then for each positive linear functional $f$ on $\mathcal{A}, f\left(x^{*} a^{*} a x\right) \leq|a|_{\infty}^{2} f\left(x^{*} x\right)$ for all $x \in \mathcal{A}$. It follows that $\pi_{f}(a)$ is bounded, and $\sup _{f}\left\|\pi_{f}(a)\right\| \leq|a|_{\infty}$. Let $\pi=$ $\sum_{f}^{\oplus} \pi_{f}$. By [12], $\pi$ represents $\mathcal{A}$ faithfully as an extended $\mathrm{C}^{*}$-algebra with common dense domain $\mathcal{D}(\pi)$ in the Hilbert space $\mathcal{H}=\bigoplus_{f} \mathcal{H}_{f}$. Then for all $\xi=\left(\xi_{f}\right) \in \mathcal{D}(\pi)$ we have

$$
\|\pi(a) \xi\|^{2}=\sum_{f}\left\|\pi_{f}(a) \xi_{f}\right\|^{2} \leq\left(\sup _{f}\left\|\pi_{f}(a)\right\|\right) \sum_{f}\left\|\xi_{f}\right\|^{2} \leq|a|_{\infty}^{2}\|\xi\|^{2} .
$$

Thus $\pi(a)$ is bounded, and $\|\pi(a)\| \leq|a|_{\infty}$. Since $\pi\left\lceil_{\mathcal{A}\left(\mathbf{B}_{0}\right)}\right.$ is one to one, we have $\|a\|_{\mathbf{B}_{0}}=\|\pi(a)\|$. Thus $\mathcal{A}\left(\mathbf{B}_{0}\right)=\mathcal{A}^{u b}$ and $\|\cdot\|_{\mathbf{B}_{0}}=|\cdot|_{\infty}$.

A complete locally m-convex $*$-algebra $\mathcal{A}$ is a complete topological *-algebra $\mathcal{A}$ whose topology is determined by a family of $*$-invariant submultiplicative seminorms. By [6, Proposition 3.2], every continuous positive linear functional on $\mathcal{A}$ is admissible. The following is an analogue of this result for quasi-weights.

Proposition 3.15. Let $\varphi$ be a quasi-weight on $\mathcal{P}\left(\mathfrak{N}_{\varphi}\right)$ in a locally m-convex $*$ algebra $\mathcal{A}$. If $\varphi$ is continuous on $\mathcal{D}(\varphi)$, then $\varphi$ is admissible.

Proof. This follows from Proposition 3.5 (2).

The following can be easily proved.

Proposition 3.16. Let $\mathcal{M}$ be an $O^{*}$-algebra on a dense subspace $\mathcal{D}$ of a Hilbert space $\mathcal{H}$. Let $\mathcal{M}_{b}=\{T \in \mathcal{M}: T$ is bounded $\}$. Then $\mathcal{M}^{u b}=\mathcal{M}_{b}$, and $|\cdot|_{\infty}=\|\cdot\|$ (the operator norm on $\mathcal{M}$ ).

We do not know whether in the above $\mathcal{M}^{b}=\mathcal{M}^{u b}$ or not.

\section{The CASE $\mathcal{A}=\mathcal{A}_{0}$ IN A LOCALly CONVEX $*$-ALGEBRA}

Theorem 3.12 suggests that from the point of view of admissibility, it is important to characterize locally convex $*$-algebras $\mathcal{A}$ such that $\mathcal{A}=\mathcal{A}_{0}$. In this section, we consider this problem. An element $x$ of $\mathcal{A}$ is quasi-regular (resp. quasi-invertible) if $(1-x)$ has inverse belonging to the unitization $\mathcal{A}_{1}$ of $\mathcal{A}$ (resp. belonging to $\left.\left(\mathcal{A}_{0}\right)_{1}\right)$. Let $\mathcal{A}^{q r}$ (resp. $\mathcal{A}^{q i}$ ) be the set of all quasi-regular (resp. quasi-invertible) elements of $\mathcal{A}$. For $x \in \mathcal{A}^{q r}, x^{q}$ denotes the quasi-inverse of $x$, i.e. $\left(1-x^{q}\right)(1-x)=$ $(1-x)\left(1-x^{q}\right)=1$.

Lemma 4.1. Let $\mathcal{A}$ be pseudo-complete. Let $p$ be a continuous seminorm on $\mathcal{A}$. The following are equivalent. 
(1) $\{x \in \mathcal{A}: p(x)<1\} \subset \mathcal{A}^{q i}$.

(2) $\beta(x) \leq p(x)$ for all $x \in \mathcal{A}$.

Proof. (1) $\Rightarrow(2)$ Take $x \in \mathcal{A}, \lambda \in \mathbb{C}$. Let $|\lambda|>p(x)$. Then by (1), $\lambda^{-1} x$ is quasiinvertible, and so $\lambda 1-x$ has inverse in $\left(\mathcal{A}_{0}\right)_{1}$. From [1, Theorem 3.1], it follows that there exist $\mathbf{B} \in \mathcal{B}$ and a neighbourhood $N$ of $\lambda$ such that for each $\mu \in N, \mu 1-x$ is investible, $R_{\mu}=(\mu 1-x)^{-1} \in \mathcal{A}(\mathbf{B})_{1}$ and $R$ is holomorphic at $\lambda$ in the sense of norm convergence in $\mathcal{A}(\mathbf{B})_{1}$. Hence $|\lambda|^{-(n+1)} x^{n} \rightarrow 0$ in the norm of $\mathcal{A}(\mathbf{B})_{1}$, which implies, by $[1$, Proposition 2.14], that $\beta(x) \leq|\lambda|$. Hence $\beta(x) \leq p(x)$.

$(2) \Rightarrow(1)$ Let $x \in \mathcal{A}$. Let $\delta>0$ be such that $p(x)<\delta<1$. Since $\beta(x) \leq p(x)<$ $\delta$, there exists $\mathbf{B} \in \mathcal{B}$ such that $x \in \mathcal{A}(\mathbf{B})$ and $\|x\|_{\mathbf{B}}<\delta$. Then we have

$$
\left\|-\sum_{k=n}^{m} x^{k}\right\|_{\mathbf{B}} \leq \sum_{k=n}^{m}\|x\|_{\mathbf{B}}^{k}<\sum_{k=n}^{m} \delta^{k} .
$$

Since $\left(\mathcal{A}(\mathbf{B}),\|\cdot\|_{\mathbf{B}}\right)$ is complete, it follows that $-\sum_{k=1}^{\infty} x^{k}$ converges to $y$ in $\mathcal{A}(\mathbf{B})$. Clearly, $y=x^{q}$. Thus $x \in \mathcal{A}^{q i}$.

Theorem 4.2. Let $\mathcal{A}$ be a pseudo-complete locally convex $*$-algebra. Consider the following statements.

(1) $\mathcal{A}$ has continuous quasi-inverse, i.e. there exists a neighbourhood $U$ of o such that $U \subset \mathcal{A}^{q r}$ and the quasi-inversion $x \rightarrow x^{q}$ is continuous at $o$.

(2) $\mathcal{A}^{q r}$ is open.

(3) There exists a continuous seminorm $p$ on $\mathcal{A}$ such that $\beta(x) \leq p(x)$ for all $x \in \mathcal{A}$.

(4) $\mathcal{A}=\mathcal{A}_{0}$.

Then the following implications hold.

$$
(1) \Rightarrow \underset{(1)}{\stackrel{(2)}{\mathbb{1}}} \Rightarrow(4) \text {. }
$$

If the multiplication of $\mathcal{A}$ is jointly continuous (e.g. $\mathcal{A}$ is a Fréchet *-algebra), then the above statements (1), (2) and (3) are equivalent.

Proof. $(2) \Leftrightarrow(3)$ follows from Lemma 4.1.

$(1) \Rightarrow(3)$. By (1), there exists a continuous seminorm $p$ on $\mathcal{A}$ such that $\{x \in \mathcal{A}: p(x)<1\} \subset \mathcal{A}^{q r}$. Then $x(x-\lambda 1)^{-1}$ is a holomorphic function on $\{\lambda \in \mathbb{C}:|\lambda|>p(x)\}$ and

$$
\lambda^{n-1} x(x-\lambda 1)^{-1}=-\left\{\lambda^{n-2} x+\cdots+x^{n-1}+\frac{x^{n}}{\lambda}\right\}-\frac{x^{n+1}}{\lambda^{2}}\left(1-\frac{x}{\lambda}\right)^{-1}
$$

for all $n \in \mathbb{N}$. By the residue theorem,

$$
x^{n}=-\frac{1}{2 \pi i} \int_{|\lambda|=\delta} \lambda^{n-1} x(x-\lambda 1)^{-1} d \lambda
$$

for each $\delta>p(x)$. Hence for each continuous seminorm $q$ on $\mathcal{A}$, we have $q\left(x^{n}\right) \leq$ $\alpha \delta^{n-1}$ ( $\alpha$ is a constant), and so $\varlimsup_{n \rightarrow \infty} q\left(x^{n}\right)^{\frac{1}{n}} \leq \delta$. Hence it follows from [1, Proposition 2.18] that $\beta(x)=\sup _{q} \varlimsup_{n \rightarrow \infty} q\left(x^{n}\right)^{\frac{1}{n}} \leq p(x)$.

$(3) \Rightarrow(4)$ is trivial. 
Now suppose that the multiplication in $\mathcal{A}$ is jointly continuous. We show that $(3) \Rightarrow(1)$. By the equivalence of (2) and (3), we have

$$
U \equiv\{x \in \mathcal{A}: p(x)<1\} \subset \mathcal{A}^{q i} \subset \mathcal{A}^{q r}
$$

and $x^{q}=-\sum_{k=1}^{\infty} x^{k}$ for each $x \in U$. Let $q$ be any continuous seminorm on $\mathcal{A}$ and let $x \in U$. Let $p(x)<\delta<1$. Since $\beta(x) \leq p(x)$, there exists $n_{1} \in \mathbb{N}$ such that $q\left(x^{n}\right)^{\frac{1}{n}}-\frac{1}{n}<\delta$ for all $n \geq n_{1}$. Let $\varepsilon>0$. Let $n_{2} \in \mathbb{N}$ be such that $\sum_{n=n_{2}}^{\infty} \delta^{n}<\varepsilon$. Take $n_{0}=\max \left(n_{1}, n_{2}\right)$. Then we have

$$
q\left(-\sum_{k=1}^{\infty} x^{k}\right) \leq \sum_{k=1}^{n_{0}-1} q\left(x^{k}\right)+\sum_{k=n_{0}}^{\infty} \delta^{k} \leq \sum_{k=1}^{n_{0}-1} q\left(x^{k}\right)+\varepsilon .
$$

This implies, by the joint continuity of multiplication, that $x \rightarrow x^{q}$ is continuous. This completes the proof.

Corollary 4.3. Let $\mathcal{A}$ be a complete locally m-convex *-algebra. If $\mathcal{A}$ is a $Q$ algebra (i.e., $\mathcal{A}^{q r}$ is open), then every quasi-weight in $\mathcal{A}$ is admissible.

\section{Approximate admissibility}

In this section, we define the notion of approximately admissible quasi-weights, and characterize them. Throughout this section, let $\mathcal{A}$ be a $*$-algebra.

Definition 5.1. Let $\varphi$ be a quasi-weight on $\mathcal{A}$. If there exists a net $\left\{\varphi_{\alpha}\right\}$ of admissible quasi weights on $\mathcal{A}$ such that $\varphi_{\alpha} \leq \varphi, \forall \alpha$ and $\varphi\left(x^{*} x\right)=\lim _{\alpha} \varphi_{\alpha}\left(x^{*} x\right), \forall x \in$ $\mathfrak{N}_{\varphi}$, then $\varphi$ is said to be approximately admissible. If there do not exist any nonzero admissible quasi-weights $\psi$ on $\mathcal{A}$ such that $\psi \leq \varphi$, then $\varphi$ is called strictly inadmissible.

Lemma 5.2. Let $\varphi$ be a quasi-weight on $\mathcal{A}$. Let $\psi$ be an admissible quasi-weight on $\mathcal{A}$ such that $\psi \leq \varphi$. Then there exists an element $K \in \pi_{\varphi}(\mathcal{A})_{\mathrm{w}}^{\prime}$ such that

(i) $0 \leq K \leq 1$,

(ii) $\pi_{\varphi}{ }^{*}(a) K^{\frac{1}{2}} \in B\left(\mathcal{H}_{\varphi}\right)$ for each $a \in \mathcal{A}$,

(iii) $\psi\left(x^{*} x\right)=\left\langle K \lambda_{\varphi}(x), \lambda_{\varphi}(x)\right\rangle$ for each $x \in \mathfrak{N}_{\varphi}$.

Proof. Take $K=K_{\varphi, \psi}^{*} K_{\varphi, \psi}$ of Lemma 2.4. Since $\psi$ is admissible, it follows that

$$
\left\|K^{\frac{1}{2}} \pi_{\varphi}(a) \lambda_{\varphi}(x)\right\|^{2}=\psi\left(x^{*} a^{*} a x\right) \leq p_{\psi}(a)^{2} \psi\left(x^{*} x\right) \leq p_{\psi}(a)^{2}\left\|\lambda_{\varphi}(x)\right\|^{2}
$$

for all $x \in \mathfrak{N}_{\varphi}$ and all $a \in \mathcal{A}$, which implies that $\overline{K^{\frac{1}{2}} \pi_{\varphi}(a)} \in \mathcal{B}\left(\mathcal{H}_{\varphi}\right)$. Hence, we have $\pi_{\varphi}{ }^{*}(a) K^{\frac{1}{2}} \in B\left(\mathcal{H}_{\varphi}\right), \forall a \in \mathcal{A}$. The rest are trivial.

Lemma 5.3. Let $\varphi$ be a quasi-weight on $\mathcal{A}$. The following statements hold.

(1) Suppose $\varphi$ is admissible. Then every quasi-weight $\psi$ on $\mathcal{A}$ with $\psi \prec \varphi$ is admissible.

(2) Suppose $\varphi$ is a regular, approximately admissible quasi-weight on $\mathcal{A}$ such that $\pi_{\varphi}(\mathcal{A})_{\mathrm{w}}^{\prime}$ is a von Neumann algebra. Then every quasi-weight $\psi$ on $\mathcal{A}$ with $\psi \prec \varphi$ is approximately admissible.

Proof. (1) Suppose that $\varphi$ is admissible. By Lemma 5.2, we have

$$
\psi\left(x^{*} a^{*} a x\right)=\left\|K^{\frac{1}{2}} \pi_{\varphi}(a) \lambda_{\varphi}(x)\right\|^{2} \leq\left\|\overline{\pi_{\varphi}(a)}\right\|^{2} \psi\left(x^{*} x\right)
$$

for all $a \in \mathcal{A}$ and $x \in \mathfrak{N}_{\varphi}=\mathfrak{N}_{\psi}$, which implies that $\psi$ is admissible. 
(2) Since $\varphi$ is approximately admissible, there exists a net $\left\{\varphi_{\alpha}\right\}$ of admissible quasi-weights on $\mathcal{A}$ such that $\varphi_{\alpha} \leq \varphi, \forall \alpha$ and $\lim _{\alpha} \varphi_{\alpha}=\varphi$. By Lemma 5.2, for each $\alpha$, there is $K_{\alpha} \in \pi_{\varphi}(\mathcal{A})_{\mathrm{w}}^{\prime}$ such that

$$
\begin{aligned}
& 0 \leq K_{\alpha} \leq I, \\
& \varphi_{\alpha}\left(y^{*} x\right)=\left\langle K_{\alpha} \lambda_{\varphi}(x), \lambda_{\varphi}(y)\right\rangle, \forall x, y \in \mathfrak{N}_{\varphi}, \\
& \left\|\pi_{\varphi}{ }^{*}(a) K_{\alpha}^{\frac{1}{2}} \xi\right\| \leq k_{\alpha}\left\|K_{\alpha}^{\frac{1}{2}} \xi\right\|, \quad \forall a \in \mathcal{A}, \xi \in \mathcal{H}_{\varphi} .
\end{aligned}
$$

Since $0 \leq K_{\alpha} \leq I$ and $\lim _{\alpha} \varphi_{\alpha}\left(x^{*} x\right)=\varphi\left(x^{*} x\right), \forall x \in \mathfrak{N}_{\varphi}$, it follows that $K_{\alpha} \rightarrow I$ weakly. Since $\psi \prec \varphi$, Lemma 2.4 implies that there is an element $H$ of $\pi_{\varphi}(\mathcal{A})_{\mathrm{w}}^{\prime}$ such that $0 \leq H \leq I$ and $\psi\left(y^{*} x\right)=\left\langle H \lambda_{\varphi}(x), \lambda_{\varphi}(y)\right\rangle$ for all $x, y$ in $\mathfrak{N}_{\varphi}$. We now put

$$
\psi_{\alpha}=\varphi_{H^{\frac{1}{2}} K_{\alpha} H^{\frac{1}{2}}}
$$

Since $\pi_{\varphi}(\mathcal{A})_{\mathrm{w}}^{\prime}$ is a von Neumann algebra, we have $H^{\frac{1}{2}} K_{\alpha} H^{\frac{1}{2}} \in \pi_{\varphi}(\mathcal{A})_{\mathrm{w}}^{\prime}, 0 \leq$ $H^{\frac{1}{2}} K_{\alpha} H^{\frac{1}{2}} \leq I$. Hence it follows from Lemma 2.7 that $\psi_{\alpha}$ is a quasi-weight on $\mathcal{A}$ such that $\mathfrak{N}_{\psi_{\alpha}}=\mathfrak{N}_{\psi}=\mathfrak{N}_{\varphi}$ and $\psi_{\alpha} \leq \psi$ (that is, $\psi_{\alpha} \prec \psi$ ). Furthermore, we have, for each $a \in \mathcal{A}$ and $x \in \mathfrak{N}_{\varphi}$,

$$
\begin{aligned}
\psi_{\alpha}\left(x^{*} a^{*} a x\right) & =\left\|K_{\alpha}^{\frac{1}{2}} H^{\frac{1}{2}} \pi_{\varphi}(a) \lambda_{\varphi}(x)\right\|^{2} \\
& =\left\|\pi_{\varphi}{ }^{*}(a) K_{\alpha}^{\frac{1}{2}} H^{\frac{1}{2}} \lambda_{\varphi}(x)\right\|^{2} \\
& \leq k_{a}^{2}\left\|K_{\alpha}^{\frac{1}{2}} H^{\frac{1}{2}} \lambda_{\varphi}(x)\right\|^{2} \\
& =k_{a}^{2} \psi_{\alpha}\left(x^{*} x\right)
\end{aligned}
$$

and

$$
\begin{aligned}
\lim _{\alpha} \psi_{\alpha}\left(x^{*} x\right) & =\lim _{\alpha}\left\langle K_{\alpha} H^{\frac{1}{2}} \lambda_{\varphi}(x), H^{\frac{1}{2}} \lambda_{\varphi}(x)\right\rangle \\
& =\left\langle H^{\frac{1}{2}} \lambda_{\varphi}(x), H^{\frac{1}{2}} \lambda_{\varphi}(x)\right\rangle \\
& =\psi\left(x^{*} x\right),
\end{aligned}
$$

which implies that $\psi$ is approximately admissible. This completes the proof.

Lemma 5.4. Let $\varphi$ be a regular approximately admissible quasi-weight on $\mathcal{A}$ such that $\pi_{\varphi}(\mathcal{A})_{\mathrm{w}}^{\prime}$ is a von Neumann algebra. Then there exists a net $\left\{E_{\alpha}\right\}_{\alpha \in \Lambda}$ of mutually orthogonal projections in $\pi_{\varphi}(\mathcal{A})_{\mathrm{w}}^{\prime}$ such that $\sum_{\alpha} E_{\alpha}=I$ and $\pi_{\varphi}{ }^{*}(a) E_{\alpha} \in \mathcal{B}\left(\mathcal{H}_{\varphi}\right)$ for each $a \in \mathcal{A}$ and $\alpha \in \Lambda$.

Proof. Let $\left\{E_{\alpha}\right\}$ be a maximal set of mutually orthogonal projections in $\pi_{\varphi}(\mathcal{A})_{\mathrm{w}}^{\prime}$ such that $\pi_{\varphi}{ }^{*}(a) E_{\alpha} \in \mathcal{B}\left(\mathcal{H}_{\varphi}\right)$ for each $a \in \mathcal{A}$ and each $\alpha \in \Lambda$. Suppose $E=$ $I-\sum_{\alpha} E_{\alpha} \neq 0$. We put $\varphi_{E}\left(x^{*} x\right)=\left\langle E \lambda_{\varphi}(x), \lambda_{\varphi}(x)\right\rangle, x \in \mathfrak{N}_{\varphi}$. By Lemma 5.3, $\varphi_{E}$ is an approximately admissible quasi-weight on $\mathcal{A}$ such that $\mathfrak{N}_{\varphi_{E}}=\mathfrak{N}_{\varphi}$ and $\varphi_{E} \leq \varphi$. Hence there exists a non-zero admissible quasi-weight $\psi$ on $\mathcal{A}$ such that $\psi \leq \varphi_{E}$. By Lemma 5.2, there exists $K \in \pi_{\varphi}(\mathcal{A})_{\mathrm{w}}^{\prime}$ such that $0 \leq K \leq I, \pi_{\varphi}{ }^{*}(x) K^{\frac{1}{2}} \in \overline{\mathcal{B}}\left(\mathcal{H}_{\varphi}\right)$ and $\psi\left(y^{*} x\right)=\left\langle K \lambda_{\varphi}(x), \lambda_{\varphi}(y)\right\rangle, \forall x, y$ in $\mathfrak{N}_{\varphi}$. Let $K=\int_{0}^{1} \lambda d F(\lambda)$ be the spectral resolution of $K$. Since $K \neq 0$, there exists a $\lambda_{0} \in \mathbb{R}, 0<\lambda_{0}<1$, such that $F=I-F\left(\lambda_{0}\right) \neq 0$ and $\lambda_{0} F \leq K$. Since $\mathfrak{N}_{\varphi_{F}}=\mathfrak{N}_{\varphi_{K}}=\mathfrak{N}_{\varphi}, \varphi_{F} \leq \frac{1}{\lambda_{0}} \varphi_{K}=\frac{1}{\lambda_{0}} \psi$ and $\psi$ is admissible, it follows from Lemma 5.3 that $\varphi_{F}$ is admissible. Further, since $\left\|F \lambda_{\varphi}(x)\right\| \leq \frac{1}{\sqrt{\lambda_{0}}}\left\|E \lambda_{\varphi}(x)\right\|$ for all $x \in \mathfrak{N}_{\varphi}$, we have $F \leq E$. This contradicts the maximality of $\left\{E_{\alpha}\right\}$. Hence, $\sum_{\alpha} E_{\alpha}=I$. 
Theorem 5.5. Let $\varphi$ be a regular quasi-weight on $\mathcal{P}\left(\mathfrak{N}_{\varphi}\right)$ in $\mathcal{A}$ such that $\pi_{\varphi}(\mathcal{A})_{\mathrm{w}}^{\prime} \mathcal{D}\left(\pi_{\varphi}\right) \subset \mathcal{D}\left(\pi_{\varphi}\right)$. Then there exists a projection $E$ in $\pi_{\varphi}(\mathcal{A})_{\mathrm{w}}^{\prime}$ such that $\varphi_{E}$ is approximately admissible and $\varphi_{I-E}$ is strictly inadmissible. Further, $E$ can be taken as $E=\sum_{\alpha} E_{\alpha}$, where $\left\{E_{\alpha}\right\}$ is a family of mutually orthogonal projections in $\pi_{\varphi}(\mathcal{A})_{\mathrm{w}}^{\prime}$ such that each $\varphi_{E_{\alpha}}$ is admissible.

Proof. Let $\left\{E_{\alpha}\right\}$ be a maximal set of mutually orthogonal projections in $\pi_{\varphi}(\mathcal{A})_{\mathrm{w}}^{\prime}$ such that $\varphi_{E_{\alpha}}$ is admissible for each $\alpha$. Take $E=\sum_{\alpha} E_{\alpha}$. Then $\varphi_{E}$ is approximately admissible, and (as in Lemma 5.4) $\varphi_{I-E}$ is strictly inadmissible.

Theorem 5.6. Let $\varphi$ be a regular quasi-weight on $\mathcal{P}\left(\mathfrak{N}_{\varphi}\right)$ in $\mathcal{A}$. Then the following are equivalent.

(1) $\varphi$ is approximately admissible and $\pi_{\varphi}(\mathcal{A})_{\mathrm{w}}^{\prime} \mathcal{D}\left(\pi_{\varphi}\right) \subset \mathcal{D}\left(\pi_{\varphi}\right)$.

(2) $\pi_{\varphi}=\sum_{\alpha}^{\oplus} \pi_{\alpha}$ for some set $\left\{\pi_{\alpha}\right\}$ of bounded operator representations of $\mathcal{A}$.

Proof. (1) $\Rightarrow(2)$ By Lemma 5.4 there exists a set $\left\{E_{\alpha}\right\}$ of mutually orthogonal projections in $\pi_{\varphi}(\mathcal{A})_{\mathrm{w}}^{\prime}$ such that $\sum_{\alpha} E_{\alpha}=I$ and $\pi_{\varphi}^{*}(x) E_{\alpha} \in \mathcal{B}\left(\mathcal{H}_{\varphi}\right)$ for each $x \in \mathcal{A}$. For each $\alpha$ we put $\pi_{\alpha}(x)=\pi_{\varphi}^{*}(x) E_{\alpha}, x \in \mathcal{A}$. It is easy to show that $\pi_{\alpha}$ is a bounded operator representation of $\mathcal{A}$. For each $\xi=\left(\xi_{\alpha}\right) \in \mathcal{D}\left(\sum^{\oplus} \pi_{\alpha}\right)$ and $x \in \mathcal{A}$ we have

$$
\begin{aligned}
\left\langle\pi_{\varphi}(x)^{*} \eta, \xi\right\rangle & =\sum_{\alpha}\left\langle\pi_{\varphi}(x)^{*} \eta, E_{\alpha} \xi\right\rangle \\
& =\sum_{\alpha}\left\langle\left(\pi_{\varphi}(x) E_{\alpha}\right)^{*} \eta, \xi\right\rangle \\
& =\sum_{\alpha}\left\langle\eta, \overline{\pi_{\varphi}(x) E_{\alpha}} \xi\right\rangle \\
& =\sum_{\alpha}\left\langle\eta, \pi_{\varphi}^{*}(x) E_{\alpha} \xi\right\rangle \\
& =\left\langle\eta, \pi_{\alpha}(x) \xi_{\alpha}\right\rangle
\end{aligned}
$$

for each $\eta \in \mathcal{D}\left(\pi_{\varphi}(x)^{*}\right)$, which implies $\sum^{\oplus} \pi_{\alpha} \subset \pi_{\varphi}$. Hence, we have $\pi_{\varphi}^{*} \subset$ $\left(\sum^{\oplus} \pi_{\alpha}\right)^{*}=\sum^{\oplus} \pi_{\alpha} \subset \pi_{\varphi}$, which implies $\pi_{\varphi}=\sum^{\oplus} \pi_{\alpha}$.

$(2) \Rightarrow(1)$ This follows from Lemma 5.4.

Corollary 5.7. Let $\varphi$ be a regular quasi-weight on $\mathcal{A}$. The following statements are equivalent.

(1) $\varphi$ is approximately admissible and $\pi_{\varphi}(\mathcal{A})_{\mathrm{w}}^{\prime}$ is a von Neumann algebra.

(2) $\widehat{\pi_{\varphi}}=\sum^{\oplus} \pi_{\alpha}$ for some set $\left\{\pi_{\alpha}\right\}$ of bounded operator representations of $\mathcal{A}$, where $\widehat{\pi_{\varphi}}$ is the induced extension of $\pi_{\varphi} \quad$ [21, Section 8.5].

\section{EXAMPLES AND APPLICATIONS}

6.1. Weight analogue of the Bochner-Weil-Raikov theorem: An application to abelian harmonic analysis. Let $\mathcal{A}$ be a commutative Banach *-algebra. Let $\Delta(\mathcal{A})$ denote its Gelfand space. Let $\hat{\mathcal{A}}=\left\{\varphi \in \Delta(\mathcal{A}): \varphi=\varphi^{*}\right\}$ be its Hermitian part. Here $\varphi^{*}(x)=\overline{\varphi\left(x^{*}\right)}(x \in \mathcal{A})$. The abstract Bochner-Weil-Raikov integral representation theorem states that $f$ is a representable positive linear functional on $\mathcal{A}$ iff there exists a bounded positive regular Borel measure $\mu$ on $\hat{\mathcal{A}}$ such that $f(x)=\int_{\hat{\mathcal{A}}} \hat{x}(\chi) d \mu(\chi), x \in \mathcal{A} \rightarrow \hat{x} \in C_{0}(\Delta(\mathcal{A}))$ being the Gelfand transform. The following theorem, which is essentially a reformation of [13, Theorem 21], provides a weight analogue of this. We call a quasi-weight $\varphi$ on a $*$-algebra $\mathcal{A}$ non-degenerate if $\pi_{\varphi}$ is non-degenerate in the sense that $\left[\pi_{\varphi}(\mathcal{A}) \lambda_{\varphi}\left(\mathfrak{N}_{\varphi}\right)\right]^{-}=\mathcal{H}_{\varphi}$. 
Theorem 6.1.1. Let $(\mathcal{A},\|\cdot\|)$ be a commutative Banach $*$-algebra (not necessarily having identity). Let $\left(\varphi, \mathfrak{N}_{\varphi}\right)$ be a quasi-weight in $\mathcal{A}$. Assume that $\varphi$ is nondegenerate. Then there exists a positive, not necessarily bounded, regular Borel measure $\mu$ on $\hat{\mathcal{A}}$ such that the following hold:

(i) $\varphi(x)=\int_{\hat{\mathcal{A}}} \hat{x}(\chi) d \mu(\chi)$ for all $x \in \mathcal{P}\left(\mathfrak{N}_{\varphi}\right)$.

(ii) For all $a, b$ in $\mathfrak{N}_{\varphi}$ and $x \in \mathcal{A}$, $\hat{a} \hat{b} \in L^{1}(\mu)$ and

$$
\begin{aligned}
& \varphi\left(b^{*} a\right)=\int_{\hat{\mathcal{A}}} \hat{a}(\chi) \overline{\hat{b}(\chi)} d \mu(\chi), \\
& \varphi\left(b^{*} x a\right)=\int_{\hat{\mathcal{A}}} \hat{a}(\chi) \hat{x}(\chi) \overline{\hat{b}(\chi)} d \mu(\chi) .
\end{aligned}
$$

(iii) $\pi_{\varphi}$ is unitarily equivalent to a subrepresentation of the representation $M$ of $\mathcal{A}$ on $L^{2}(\mu)$ defined by $(M(x) f)(\chi)=\hat{x}(\chi) f(\chi)$.

(iv) If $\mathcal{D}(\varphi)$ is dense in $\mathcal{A}$, then $\mu$ satisfying the above is unique and $\pi_{\varphi}$ is unitarily equivalent to $M$.

Proof. By Proposition 3.8, $\varphi$ is admissible, and since $\varphi$ is non-degenerate, $x \rightarrow$ $\overline{\pi_{\varphi}(x)}$ defines a non-degenerate bounded operator representation of $\mathcal{A}$. By Stone's theorem ([13], Theorem 10.10) there exists a spectral measure $E$ on Borel subsets of $\hat{\mathcal{A}}$ such that for all $x \in \mathcal{A}, \xi, \eta$ in $\mathcal{H}_{\varphi}$,

$$
\left.\overline{\pi_{\varphi}(x)} \xi, \eta\right\rangle=\int_{\hat{\mathcal{A}}} \hat{x}(\chi) d E_{\xi, \eta}(\chi) .
$$

Let $a \in \mathfrak{N}_{\varphi}$. Let $\mu_{a}$ be the Borel measure on $\hat{\mathcal{A}}$ defined by $d \mu_{a}=d E_{\lambda_{\varphi}(a), \lambda_{\varphi}(a)}$. Then, as in ([13], p.94), $|\hat{a}(\chi)|^{2} d \mu_{b}(\chi)=|\hat{b}(\chi)|^{2} d \mu_{a}(\chi)$ for any $a, b$ in $\mathfrak{N}_{\varphi}$. Now let $f \in C_{c}(\hat{\mathcal{A}})$. Choose a $b \in \mathcal{A}$ such that $\hat{b}(\chi) \neq 0$ for all $\chi \in \operatorname{supp} f$. This defines

$$
\mu(f)=\int_{\text {suppf }} f(\chi)|\hat{b}(\chi)|^{-2} d \mu_{b}(\chi),
$$

and the above shows that $f \rightarrow \mu(f)$ is a positive linear functional on $C_{c}(\hat{\mathcal{A}})$. Thus, there exists a positive Borel measure $\mu$ on $\hat{\mathcal{A}}$ such that

$$
\mu(f)=\int_{\hat{\mathcal{A}}} f(\chi) d \mu(\chi) .
$$

Hence, for all $a \in \mathfrak{N}_{\varphi}, d_{\mu_{a}}(\chi)=|\hat{a}(\chi)|^{2} d \mu(\chi)$. By the boundedness of $\mu_{a}$ (a consequence of the definition of spectral measure), $\left(a^{*} a\right)^{\wedge} \in L^{1}(\mu)$ for all $a \in \mathfrak{N}_{\varphi}$, and $\varphi\left(a^{*} a\right)=\left\|\lambda_{\varphi}(a)\right\|^{2}=\mu_{a}(\hat{\mathcal{A}})=\int\left(a^{*} a\right)^{\gamma}(\chi) d \mu(\chi)$. Since any product $b^{*} a(a, b$ in $\mathfrak{N}_{\varphi}$ ) is a linear combination of elements of the form $c^{*} c$ with $c \in \mathfrak{N}_{\varphi}$, it follows that $\hat{b} \hat{a} \in L^{1}(\mu)$, and (ii) holds. This immediately gives (i) also. Then (iii) and (iv) can be verified as in ([13], Theorem 21.6). Note that if $\mathcal{D}(\varphi)$ is dense in $\mathcal{A}$, then given any compact set $\mathcal{K}$ in $\hat{\mathcal{A}}$, there exists $b \in \mathcal{D}(\varphi)$ such that $\hat{b}(\chi) \neq 0$ for each $\chi \in \mathcal{K}$.

In what follows, we shall apply the above to the convolution Banach algebra $L^{1}(G)$ of a locally compact abelian group $G$. The involution of $L^{1}(G)$ is $f^{*}(g)=$ $\overline{f(-g)}(g \in G)$, and $\Delta\left(L^{1}(G)\right) \approx \widehat{G}$ (the dual group of $G$ ) by the well known identification, so that the Gelfand transform gets to be identified with Fourier transform. Note that $L^{1}(G)$ is Hermitian, so that $\Delta\left(L^{1}(G)\right)=L^{1}(G)$. 
(a) Let $\varphi$ be a positive linear functional on $L^{1}(G)$. Then there exists a bounded positive Borel measure $\nu$ on $\widehat{G}$ such that

$$
\varphi(f)=\int_{\widehat{G}} \hat{f}(\chi) d \nu(\chi) .
$$

Corollary 6.1.2. Let $\left(\varphi, \mathfrak{N}_{\varphi}\right)$ be a non-degenerate quasi-weight in $L^{1}(G)$. Then there exists a positive, not necessarily bounded, regular Borel measure $\nu$ on $\widehat{G}$ such that the following hold:

(i) For all $f \in \mathcal{P}\left(\mathfrak{N}_{\varphi}\right), \varphi(f)=\int_{\widehat{G}} \hat{f}(\chi) d \nu(\chi)$.

(ii) For all $f, g \in \mathfrak{N}_{\varphi}, \quad \hat{f} \overline{\hat{g}} \in L^{1}(\widehat{G}, \nu)$, and $\varphi\left(f * g^{*}\right)=\int_{\widehat{G}} \hat{f}(\chi) \overline{\hat{g}(\chi)} d \nu(\chi)$.

(b) The extension of the concept of Fourier transform from integrable functions to positive definite complex regular Borel measures on $G$ is a standard part of harmonic analysis. We can use the concept of weight to define the Fourier transform of certain unbounded positive definite measures.

Let $\mu$ be a positive (not necessarily bounded) measure on $G$. Suppose $\mu$ is positive definite, that is, $\int_{G} f^{*} * f d \mu \geq 0$ for each $f \in L^{1}(G)$. Then we define a weight $\varphi$ on $L^{1}(G)$ by $\varphi(f)=\int_{G} f d \mu$. Assume that $\varphi$ is non-degenerate. By Corollary 6.1 .2 , there exists a regular positive measure $\hat{\mu}$ on $\widehat{G}$ such that

$$
\int_{G}(f * h)(g) d \mu(g)=\int_{\widehat{G}} \hat{f}(\chi) \hat{h}(\chi) d \hat{\mu}(\chi) \quad\left(f, h \in \mathfrak{N}_{\varphi}\right),
$$

and

$$
\int_{G} f d \mu=\int_{\widehat{G}} \hat{f} d \hat{\mu} \quad f \in \mathcal{P}\left(L^{1}(G)\right) .
$$

This $\hat{\mu}$ may be called the Fourier transform of $\mu$. This extends the considerations of ([13], 2.7, p. 1011) to unbounded measures.

\subsection{Integration algebras, measures and tracial weights.}

Definition 6.2.1. An integration algebra $(\mathcal{A}, *, \varphi)$ consists of a $*$-algebra $\mathcal{A}$ having involution $*$ and a weight $\varphi$ on $\mathcal{P}(\mathcal{A})$ that is faithful in the sense that $\varphi\left(x^{*} x\right)=0$ implies $x=0$.

This can be regarded as a framework for (unbounded non-commutative) integration theory. Let $(\mathcal{A}, *, \varphi)$ be an integration algebra. Then $\mathfrak{N}_{\varphi}=\{x \in \mathcal{A}$ : $\left.\varphi\left(x^{*} a^{*} a x\right)<\infty, \forall a \in \mathcal{A}\right\}$ is equipped with the inner product $\langle x, y\rangle=\varphi\left(y^{*} x\right), \mathcal{H}_{\varphi}$ is the Hilbert space completion of $\mathfrak{N}_{\varphi}$, and $\pi_{\varphi}$ is the closure of the left regular representation $\pi_{0}$ of $\mathcal{A}$ having $\mathcal{D}\left(\pi_{0}\right)=\mathfrak{N}_{\varphi}$ in $\mathcal{H}_{\varphi}, \pi_{0}(a) x=a x\left(a \in \mathcal{A}, x \in \mathfrak{N}_{\varphi}\right)$. We discuss below the admissibility and approximate admissibility in some examples.

(A) Measures and integrals. Let $(X, \Sigma, \mu)$ be a $\sigma$-finite measure space with positive measure $\mu$. Let $\mathcal{A}$ be a $*$-algebra of $\mu$-measurable functions on $X$. Then $\varphi(f)=$ $\int_{X} f d \mu$ defines a weight on $\mathcal{A}$.

(A1) Take $\mathcal{A}=\mathcal{M}(X, \mu)$, the set of all measurable functions. Then $\mathfrak{N}_{\varphi}^{0}=$ $L^{2}(\mu), \mathfrak{N}_{\varphi}=\left\{g \in \mathcal{M}(X, \mu): \int|f g|^{2} d \mu<\infty\right.$ for all $\left.f \in \mathcal{M}(X, \mu)\right\}=\{o\}$ if $X$ is atom free. The GNS-representation $\pi_{\varphi}$ is trivial in this case. This is to be expected in the light of the fact that when $X$ is atom free, $\mathcal{M}(X, \mu)$ admits no non-zero positive linear functional. 
(A2) Let $\mathcal{A}=L_{\text {loc }}^{\infty}(\mu)=\{f \in \mathcal{M}(X, \mu): f$ is essentially bounded on every set of finite measure $\}$.

Claims. (i) $\varphi$ is admissible iff $\mu$ is a finite measure,

(ii) $\varphi$ is approximately admissible.

Proof. Let $L_{c}^{\infty}(\mu)=\left\{f \in L^{\infty}(\mu): f=0\right.$ outside a set of finite measure $\}$. Then $L_{c}^{\infty}(\mu) \subset \mathfrak{N}_{\varphi}=\left\{f \in L_{l o c}^{\infty}(\mu): a f \in L^{2}(\mu)\right.$ for all $\left.a \in L_{\text {loc }}^{\infty}(\mu)\right\}$, and since $1 \in$ $L_{\text {loc }}^{\infty}(\mu), \mathfrak{N}_{\varphi} \subset L^{2}(\mu)$. Thus $L_{c}^{\infty}(\mu) \subset \mathfrak{N}_{\varphi} \subset L^{2}(\mu)$. Further, $N_{\varphi}=\{o\}, \mathcal{D}(\varphi)=$ span $\mathfrak{N}_{\varphi}^{*} \mathfrak{N}_{\varphi}=L_{l o c}^{\infty}(\mu) \cap L^{1}(\mu)$. Since $L_{c}^{\infty}(\mu)$ is dense in $L^{2}(\mu)$, it follows that $\mathcal{H}_{\varphi}=L^{2}(\mu)$, and $\pi_{\varphi}(a) f=a f, \forall a \in L_{l o c}^{\infty}(\mu), \forall f \in \mathcal{D}\left(\pi_{\varphi}\right)$. Now let $\varphi$ be admissible. Then $\overline{\pi_{\varphi}(a)} \in \mathcal{B}\left(L^{2}(\mu)\right), \forall a \in L_{l o c}^{\infty}(\mu)$. Hence af $\in L^{2}(\mu), \forall f \in L^{2}(\mu)$. Hence $a \in L^{\infty}(\mu)$. Thus $L_{l o c}^{\infty}(\mu)=L^{\infty}(\mu)$, showing that $\mu$ is finite. This proves (i). Now choose $A_{n} \in \Sigma, A_{n} \uparrow, \mu\left(A_{n}\right)<\infty$ and $X=\bigcup_{n=1}^{\infty} A_{n}$. Then $\varphi_{n}(f)=\int_{A_{n}} f d \mu$ defines an admissible positive linear functional on $L_{l o c}^{\infty}(\mu)$ such that $\varphi_{n} \leq \varphi$ and $\varphi=\sup \varphi_{n}$. This proves (ii).

(A3) Let $X$ be a non-compact locally compact $\sigma$-compact space. Let $\varphi$ be a positive linear functional on $C_{c}(X)$, hence given by a positive Radon measure (locally finite) $\mu$ on $X$. Given $h \geq 0$ in $C(X)$, choose $h_{n} \in C_{c}(X), h_{n} \uparrow h$. Then $\varphi(h)=\lim \varphi\left(h_{n}\right)=\lim _{n} \int h_{n} d \mu$ defines a faithful weight on $C(X)$.

Claims. (i) $\varphi$ is an admissible weight on $C(X)$ iff $\varphi$ extends as a (necessarily admissible) positive linear functional on $C(X)$ iff $\mu$ has compact support.

(ii) $\varphi$ is approximately admissible.

This can be proved as in the previous case.

(B) Traces and integrals. Let $\mathcal{D}$ be a dense subspace in a separable Hilbert space $\mathcal{H}$ and $\left\{\xi_{n}\right\}$ an orthonormal basis of $\mathcal{H}$ contained in $\mathcal{D}$. Let $\mathcal{M}$ be a closed $\mathrm{O}^{*}$-algebra on $\mathcal{D}$ with the identity operator $I$, and define a faithful (tracial) weight on $\mathcal{P}(\mathcal{M})$ by

$$
\varphi\left(X^{\dagger} X\right)=\sum_{n}\left\|X \xi_{n}\right\|^{2}=\operatorname{tr} X^{\dagger} X, X \in \mathcal{M}
$$

Then $(\mathcal{M}, \varphi)$ is an integration algebra. Let $\mathcal{H} \otimes \overline{\mathcal{H}}\left(=C^{2}(\mathcal{H})\right)$ be the $*$-algebra of all Hilbert-Schmidt operators on $\mathcal{H}$. Now $\mathfrak{N}_{\varphi}=\{X \in \mathcal{M}: \overline{A X} \in \mathcal{H} \otimes \overline{\mathcal{H}}, \forall A \in \mathcal{M}\}$, an inner product space with $\langle X, Y\rangle=\operatorname{tr} Y^{\dagger} X$; let $\mathcal{H}_{\varphi}$ be its completion in the norm $\|X\|_{2} \equiv\left(\sum_{n}\left\|X \xi_{n}\right\|^{2}\right)^{\frac{1}{2}}$. Then $\pi_{\varphi}$ is the closure of $\pi_{0}$, where $\pi_{0}(A) X=$ $A X\left(A \in \mathcal{M}, X \in \mathfrak{N}_{\varphi}\right)$. Now let $\left(\sigma, \mathcal{D}(\sigma), \mathcal{H}_{\sigma}\right)$ be the $*$-representation of $\mathcal{A}$ defined as:

$$
\begin{aligned}
& \mathcal{H}_{\sigma}=\mathcal{H} \otimes \overline{\mathcal{H}} \text { regarded as Hilbert space, } \\
& \mathcal{D}(\sigma)=\{T \in \mathcal{H} \otimes \overline{\mathcal{H}} ; T \mathcal{H} \subset \mathcal{D} \text { and } A T \in \mathcal{H} \otimes \overline{\mathcal{H}}, \forall A \in \mathcal{M}\}, \\
& \sigma(A) T=A T, \quad A \in \mathcal{M}, T \in \mathcal{D}(\sigma) .
\end{aligned}
$$

Then $\left(\sigma, \mathcal{D}(\sigma), \mathcal{H}_{\sigma}\right)$ is a closed $*$-representation of $\mathcal{A}$. Since $I \in \mathcal{M}, \mathfrak{N}_{\varphi} \hookrightarrow \mathcal{H} \otimes \overline{\mathcal{H}}$ via $X \longrightarrow \bar{X}$. Hence, $\mathcal{H}_{\varphi}$ is regarded as a closed $\sigma$-invariant subspace of $\mathcal{H} \otimes \overline{\mathcal{H}}$, and $\pi_{\varphi}$ is a $*$-subrepresentation of $\sigma$; in fact, $\pi_{\varphi} \cong\left[\sigma\left\lceil\mathfrak{N}_{\varphi}\right]^{-}\right.$, the closure of the restriction of $\sigma$ on $\mathfrak{N}_{\varphi}$. The following cases arise: 
(B1) Let $\mathcal{M}$ be an $\mathrm{O}^{*}$-algebra such that $\mathcal{M}_{b}(\equiv\{X \in \mathcal{M}: \bar{X} \in B(\mathcal{H})\})=\mathbb{C} I$. Then $\mathfrak{N}_{\varphi}^{0} \equiv\left\{X \in \mathcal{M} ; \varphi\left(X^{\dagger} X\right)<\infty\right\}=\{0\}$.

(B2) Proposition 6.2.2. Let $\mathcal{M}$ be an $O^{*}$-algebra consisting of operators commuting with $\left\{\xi_{n} \otimes \overline{\xi_{n}}: n \in \mathbb{N}\right\}$, where $(\xi \otimes \bar{\eta}) \zeta \equiv\langle\zeta, \eta\rangle \xi(\xi, \eta, \zeta \in \mathcal{H})$. Then $\varphi$ is approximately admissible.

Proof. We put

$$
\varphi_{n}(X)=\left\langle X \xi_{n}, \xi_{n}\right\rangle, \quad n \in \mathbb{N}, X \in \mathcal{M} .
$$

Then since

$$
\begin{aligned}
\varphi_{n}\left(X^{\dagger} A^{\dagger} A X\right) & =\left\|A X \xi_{n}\right\|^{2} \\
& =\left\|A X\left(\xi_{n} \otimes \overline{\xi_{n}}\right)\right\|_{2}{ }^{2} \\
& =\left\|A\left(\xi_{n} \otimes \overline{\xi_{n}}\right) X\right\|_{2}{ }^{2} \\
& =\left\|A \xi_{n} \otimes \overline{X^{\dagger} \xi_{n}}\right\|_{2}{ }^{2} \\
& =\left\|A \xi_{n}\right\|^{2}\left\|X^{\dagger} \xi_{n}\right\|^{2} \\
& =\left\|A \xi_{n}\right\|^{2}\left\|X^{\dagger}\left(\xi_{n} \otimes \overline{\xi_{n}}\right)\right\|_{2}{ }^{2} \\
& =\left\|A \xi_{n}\right\|^{2}\left\|X \xi_{n}\right\|^{2} \\
& =\left\|A \xi_{n}\right\|^{2} \varphi_{n}\left(X^{\dagger} X\right)
\end{aligned}
$$

for all $A, X \in \mathcal{M}$, it follows that $\varphi_{n}$ is an admissible positive linear functional on $\mathcal{M}$ and $\varphi\left(X^{\dagger} X\right)=\sum_{n} \varphi_{n}\left(X^{\dagger} X\right)$ for all $X \in \mathcal{M}$.

(B3) Proposition 6.2.3. Let $\mathcal{M}$ be a self-adjoint $O^{*}$-algebra on $\mathcal{D}$. Suppose $\mathcal{M}_{\mathrm{w}}^{\prime}=\mathbb{C} I$ and $\pi_{\varphi}$ is self-adjoint. Then the following statements hold:

(1) Either $\varphi$ is admissible or $\varphi$ is strictly inadmissible.

(2) $\varphi$ is admissible iff $\mathcal{M}$ consists of bounded operators.

Proof. By [15, Lemma 5.3], $\sigma$ is a self-adjoint representation of $\mathcal{M}$, so that $\sigma(\mathcal{M}) \equiv$ $\{\sigma(A) ; A \in \mathcal{M}\}$ is a self-adjoint $\mathrm{O}^{*}$-algebra in $\mathcal{H} \otimes \overline{\mathcal{H}}$ having domain $\mathcal{D}(\sigma)$. Further,

$$
\sigma(\mathcal{M})_{\mathrm{w}}^{\prime}=\pi^{\prime}(\mathcal{B}(\mathcal{H})) \equiv\left\{\pi^{\prime}(K): K \in \mathcal{B}(\mathcal{H})\right\}
$$

where $\pi^{\prime}(K) T=T K, T \in \mathcal{H} \otimes \overline{\mathcal{H}}$. Since $\pi_{\varphi}$ is a self-adjoint subrepresentation of $\sigma$, by [23, Theorem 4.7] there exists a projection $P=\pi^{\prime}(K) \in \sigma(\mathcal{M})_{\mathrm{w}}^{\prime}$ such that $\pi_{\varphi}(A)=\sigma(A) \pi^{\prime}(K), A \in \mathcal{M}$, for some $K \in \mathcal{B}(\mathcal{H})$, and $\pi^{\prime}(K) T=\pi^{\prime}(K) \pi^{\prime}(K) T$, $\forall T \in \mathcal{H} \otimes \overline{\mathcal{H}}$. Thus $T K^{2} \xi=T K \xi, \forall T \in \mathcal{H} \otimes \overline{\mathcal{H}}, \forall \xi \in \mathcal{H}$. Taking $T=\xi_{n} \otimes \overline{\xi_{n}}$, we get $\left\langle K^{2} \xi, \xi_{n}\right\rangle=\left\langle K \xi, \xi_{n}\right\rangle, \forall n \in \mathbb{N}$, and hence $K^{2}=K$. Similarly, $K^{*}=K$. Thus $K$ is a projectoin. Now suppose $\varphi$ is admissible. Then since $\sigma(A) \pi^{\prime}(K)$ is unitarily equivalent to $\pi_{\varphi}(A)$ for each $A \in \mathcal{M}$, there exists a $\gamma>0$ such that

$$
\left\|\sigma(A) \pi^{\prime}(K) T\right\|_{2}{ }^{2}=\left\|\pi_{\varphi}(A) T\right\|_{2}{ }^{2} \leq \gamma\|T\|_{2}{ }^{2}
$$

for all $T \in \mathcal{H} \otimes \overline{\mathcal{H}}$. Take $T=\xi \otimes \bar{\eta}$ for an arbitrary $\xi \in \mathcal{D}$ and a non-zero $\eta \in K \mathcal{H}$. Suppose that $\varphi$ is not strictly inadmissible. Then we have

$$
\begin{aligned}
\|\eta\|^{2}\|A \xi\|^{2} & =\sum_{n}\left\|\sigma(A) \pi^{\prime}(K)(\xi \otimes \bar{\eta}) \xi_{n}\right\|^{2} \\
& =\left\|\sigma(A) \pi^{\prime}(K)(\xi \otimes \bar{\eta})\right\|_{2}{ }^{2} \\
& \leq \gamma\|\xi \otimes \bar{\eta}\|_{2}{ }^{2} \\
& =\gamma\|\xi\|^{2}\|\eta\|^{2},
\end{aligned}
$$


so that $A$ is bounded. This proves (2). To prove (1), we show that if $\mathcal{M}$ contains an unbounded operator, then $\varphi$ is strictly inadmissible. Suppose that $\varphi$ is not strictly inadmissible. Then there exists an admissible quasi-weight $\psi$ in $\mathcal{M}$ such that $\psi \leq \varphi$, i.e., $\mathfrak{N}_{\varphi} \subset \mathfrak{N}_{\psi}$ and $\psi\left(X^{\dagger} X\right) \leq \varphi\left(X^{\dagger} X\right)$ for each $X \in \mathfrak{N}_{\varphi}$. By Lemma 5.2 there exists $K \in \pi_{\varphi}(\mathcal{M})_{\mathrm{w}}^{\prime}$ such that $0 \leq K \leq I, \pi_{\varphi}{ }^{*}(A) K^{\frac{1}{2}} \in B\left(\mathcal{H}_{\varphi}\right), \forall A \in \mathcal{M}$, and $\psi\left(Y^{\dagger} X\right)=\left\langle K \lambda_{\varphi}(X), \lambda_{\varphi}(Y)\right\rangle, X, Y \in \mathfrak{N}_{\varphi}$ Let $K=\int_{0}^{1} \lambda d F(\lambda)$ be the spectral resolutions. Then there exist $\lambda_{0} \in \mathbb{R}, 0<\lambda_{0}<1$, and $F \equiv I-F\left(\lambda_{0}\right) \neq 0$ with $\lambda_{0} F \leq K$. Define $\varphi_{F}\left(X^{\dagger} X\right)=\left\langle F \lambda_{\varphi}(X), \lambda_{\varphi}(X)\right\rangle, X \in \mathfrak{N}_{\varphi}$, a quasi-weight such that $\mathfrak{N}_{\varphi_{F}}=\mathfrak{N}_{\varphi_{K}}=\mathfrak{N}_{\varphi}$ and $\varphi_{F} \leq \frac{1}{\lambda_{0}} \varphi_{K} \leq \frac{1}{\lambda_{0}} \psi$. Since $\psi$ is admissible, $\varphi_{F}$ is admissible by Lemma 5.3. Now, as in the proof of (2), we can show that $\mathcal{A}$ is bounded. This completes the proof.

(C) Weighted traces.

(C1) Let $\Omega \in \mathcal{H} \otimes \overline{\mathcal{H}} \backslash \mathcal{D}(\sigma), \Omega \geq 0$. Then a quasi-weight $\left(\varphi_{\Omega}, \mathfrak{N}_{\varphi_{\Omega}}\right)$ is defined in $\mathcal{M}$ by $\Omega$ as follows:

$$
\begin{aligned}
& \mathfrak{N}_{\varphi_{\Omega}}=\left\{X \in \mathcal{M}: \Omega \mathcal{H} \subset \mathcal{D}\left(X^{\dagger^{*}}\right), X^{\dagger^{*}} \Omega \in \mathcal{D}(\sigma)\right\}, \\
& \varphi_{\Omega}\left(X^{\dagger} X\right)=\operatorname{tr}\left(\left(X^{\dagger^{*}} \Omega\right)^{*}\left(X^{\dagger^{*}} \Omega\right)\right), \quad X \in \mathfrak{N}_{\varphi_{\Omega}} .
\end{aligned}
$$

If $\sigma\left(\mathfrak{N}_{\varphi_{\Omega}}\right)$ is dense in $\mathcal{D}(\sigma)\left[t_{\sigma(\mathcal{M})}\right]$, then $\pi_{\varphi_{\Omega}}$ is unitarily equivalent to $\sigma$, in which case $\varphi_{\Omega}$ is admissible iff $\mathcal{M}$ consists of bounded operators.

(C2) Let $\Omega$ be an unbounded positive self-adjoint operator in $\mathcal{H}$. Suppose there exists a subspace $\mathcal{E}$ of $\mathcal{D} \cap \mathcal{D}(\Omega)$ such that

(i) $\mathcal{E}$ is dense in $\mathcal{D}\left[t_{\mathcal{M}}\right]$,

(ii) $\mathcal{M} \supset\{\xi \otimes \bar{\eta}: \xi, \eta \in \mathcal{E}\}$

(iii) $\Omega \mathcal{E} \subset \mathcal{D}, \Omega \mathcal{E}$ is dense in $\mathcal{H}$.

Let $\varphi_{\Omega}$ be the quasi-weight in $\mathcal{M}$ defined as

$$
\begin{aligned}
& \mathfrak{N}_{\varphi_{\Omega}}=\left\{X \in \mathcal{M}: \overline{X^{\dagger^{*}} \Omega} \in \sigma(\mathcal{M})\right\}, \\
& \varphi_{\Omega}\left(X^{\dagger} X\right)=\operatorname{tr}\left(\left(X^{\dagger^{*}} \Omega\right)^{*}\left(X^{\dagger^{*}} \Omega\right)\right), \quad X \in \mathfrak{N}_{\varphi_{\Omega}} .
\end{aligned}
$$

Proposition 6.2.4. $\varphi_{\Omega}$ is a faithful quasi-weight. If $\mathcal{M}$ contains an unbounded operator, then $\varphi_{\Omega}$ is strictly inadmissible.

Proof. Let $\left\{\xi_{n}\right\}$ be an orthonormal basis in $\mathcal{H}$ contained in $\mathcal{D}(\Omega)$. Then, $0=$ $\varphi_{\Omega}\left(X^{\dagger} X\right)=\sum_{n}\left\|X^{\dagger^{*}} \Omega \xi_{n}\right\|^{2}$ implies that $X^{\dagger^{*}} \Omega=0$ on $\mathcal{D}(\Omega)$. Since $\Omega \mathcal{E}$ is dense in $\mathcal{H}$, it follows that $X^{\dagger^{*}} \xi=0$ for all $\xi \in \mathcal{D}$. Hence $X=0$, and so $\varphi_{\Omega}$ is faithful. Now under the stated conditions, $\overline{\mathfrak{N}_{\varphi_{\Omega}}{ }^{\dagger^{*}} \Omega}$ is dense in $\left(\mathcal{D}(\sigma), t_{\sigma(\mathcal{M})}\right)$; hence $\pi_{\varphi_{\Omega}} \sim \sigma$. Further, $\varphi_{\Omega}$ is regular [3]. Thus if $\varphi_{\Omega}$ is not strictly inadmissible, then $\sigma$ admits a non-trivial bounded subrepresentation, and so there exists a projection $K$ in $\mathcal{B}(\mathcal{H})$ such that $\left\|\sigma(A) \pi^{\prime}(K) T\right\|_{2} \leq k_{A}\left\|\pi^{\prime}(K) T\right\|_{2}$ for all $T \in \mathcal{H} \otimes \overline{\mathcal{H}}$. This implies that each $A \in \mathcal{M}$ is a bounded operator.

(D) Quasi-weights and $O^{*}$-algebra approach to statistical mechanics. We consider the problem of handling the equilibrium states of an infinite system in the algebraic approach. Let $\mathcal{F}$ be the collection of all bounded open subsets of $\mathbb{R}^{3}$. For $V \in \mathcal{F}$, let $\mathcal{A}_{V}$ be the observable $*$-algebra related to the bounded region $V$. Depending on the scheme under consideration, $\mathcal{A}_{V}$ is either a $\mathrm{C}^{*}$-algebra or an $\mathrm{O}^{*}$-algebra, consisting of operators in the Fock space $\mathcal{H}_{V}$ for $V$. Let $H_{V}$ denote the Hamiltonian in $V$, a self-adjoint operator in $\mathcal{H}_{V}$, written-down explicitly from quantum mechanics. The Heisenberg dynamics in $V$ is given by the one-parameter group of $*$-automorphisms 
$t \rightarrow \alpha_{t}^{V}: \mathcal{A}_{V} \rightarrow \mathcal{A}_{V}, \alpha_{t}^{V}(A)=e^{i t H_{V}} A e^{-i t H_{V}}$. By the Gibbs postulate, the equilibrium state for the finite subsystem in $V$ is given by the formal expression

$$
\omega_{V}(A)=\frac{1}{\operatorname{tr}\left(e^{-\beta H_{V}}\right)} \operatorname{tr}\left(e^{-\beta H_{V}} A\right) \quad\left(A \in \mathcal{A}_{V}\right) .
$$

This requires $e^{-\beta H_{V}}(\beta>0)$ to be a trace class operator.

(a) When $\mathcal{A}_{V}$ is a $\mathrm{C}^{*}$-algebra, $\mathcal{A}_{V} \subset \mathcal{B}\left(\mathcal{H}_{V}\right)$, then $e^{-\beta H_{V}} A$ is of trace class for all $A \in \mathcal{A}_{V}$. Thus (i) defines a normalized positive linear functional on $\mathcal{A}_{V}$. In the abstract formalism, a normalized positive functional (on $\mathcal{A}_{V}$ ) satisfying the KMS condition with respect to $t \rightarrow \alpha_{t}^{V}$ has been proposed as the desired equilibrium in $V$.

(b) Suppose $\mathcal{A}_{V}$ is an $\mathrm{O}^{*}$-algebra. Then (i) can be interpreted as a quasi-weight $\left(\omega_{V}, \mathfrak{N}_{\omega_{V}}\right)$ in $\mathcal{A}_{V}$ having

$$
\mathfrak{N}_{\omega_{V}}=\left\{X \in \mathcal{A}_{V}: \operatorname{tr}\left(e^{-\beta H_{V}} X^{\dagger} A^{\dagger} A X\right)<\infty \text { for all } A \in \mathcal{A}_{V}\right\},
$$

and $\omega_{V}\left(X^{\dagger} X\right)=\operatorname{tr}\left(\rho_{V} X^{\dagger} X\right), \rho_{V}=e^{-\beta H_{V}} / \operatorname{tr}\left(e^{-\beta H_{V}}\right)$. In general, one takes $\left(\omega_{V}, \mathfrak{N}_{\omega_{V}}\right)$ to be any quasi-weight in $\mathcal{A}_{V}$ satisfying the KMS condition with respect to $t \rightarrow \alpha_{t}^{V}$. This interpretation is consistent with the general formalism suggested in [3] that when the observable algebra is an $\mathrm{O}^{*}$-algebra, the states are better represented by quasi-weights. (Note that positive functionals are included in quasi-weights).

Now the isotopy condition on the local net $V \rightarrow \mathcal{A}_{V}$ can be expressed by the assumption that $V_{1} \leq V_{2}$ in $\mathcal{F} \Longrightarrow \mathcal{A}_{V_{1}} \hookrightarrow \mathcal{A}_{V_{2}}$ via an injective $*$-isomorphism taking $\mathfrak{N}_{\omega_{V_{1}}} \hookrightarrow \mathfrak{N}_{\omega_{V_{2}}}$, and is implemented by an isometry $\mathcal{H}_{V_{1}} \hookrightarrow \mathcal{H}_{V_{2}}$ taking $\operatorname{Dom}\left(\mathcal{A}_{V_{1}}\right) \hookrightarrow \operatorname{Dom}\left(\mathcal{A}_{V_{2}}\right)$. The $\mathrm{O}^{*}$-algebra of local observales is $\mathcal{A}_{l}=\bigcup_{V} \mathcal{A}_{V}$. Now the passage to the infinite system (thermodynamic limit) $\operatorname{Lim}_{V \rightarrow \infty}$ involves the following problems.

(1) Existence of equilibrium given formally by $\operatorname{Lim}_{V \rightarrow \infty} \omega_{V}(\cdot)=\omega(\cdot)$ on $\mathcal{A}_{l}$.

(2) Existence of dynamics given formally by $\operatorname{Lim}_{V \rightarrow \infty} \alpha_{t}^{V}(\cdot)=\alpha_{t}(\cdot)$.

In principle, $\omega(\cdot)$ describes equilibrium iff it satisfies the KMS condition with respect to $t \rightarrow \alpha_{t}(\cdot)$. Now $\omega$ can be interpreted as quasi-weight $\left(\omega, \mathfrak{N}_{\omega}\right)$ in $\mathcal{A}_{l}$ as

$$
\mathfrak{N}_{\omega}=\left\{X \in \mathcal{A}_{l}: \operatorname{Lim}_{V \rightarrow \infty} \omega_{V}\left(X^{\dagger} A^{\dagger} A X\right) \text { exists and is finite for all } A \in \mathcal{A}_{l}\right\},
$$

$\omega: \mathcal{P}\left(\mathfrak{N}_{\omega}\right) \rightarrow[0, \infty), \omega\left(X^{\dagger} X\right)=\operatorname{Lim}_{V \rightarrow \infty} \omega_{V}\left(X^{\dagger} X\right)$. We investigate below the admissibility of $\omega$.

Let $\left(\varphi_{V}, \mathfrak{N}_{\varphi_{V}}\right)$ be a quasi-weight in $\mathcal{A}_{V}$ defined as $\mathfrak{N}_{\varphi_{V}}=\mathfrak{N}_{\omega} \cap \mathcal{A}_{V}, \varphi_{V}\left(X^{\dagger} X\right)=$ $\omega_{V}\left(X^{\dagger} X\right)$.

Proposition 6.2.5. (1) Assume that $\left(\varphi_{V}, \mathfrak{N}_{\varphi_{V}}\right)$ is admissible for all $V$ and that for each $V_{0} \in \mathcal{F}, \sup _{V>V_{0}}\left\|\pi_{\varphi_{V}}(A)\right\|<\infty\left(A \in \mathcal{A}_{V_{0}}\right)$. Then $\left(\omega, \mathfrak{N}_{\omega}\right)$ is admissible.

(2) Suppose $\left(\omega, \mathfrak{N}_{\omega}\right)$ is admissible. Further assume that $V_{1} \leq V_{2}$ implies $\rho_{V_{1}} \leq$ $\rho_{V_{2}}$. Then each $\varphi_{V}$ is admissible, and for any $V_{0} \in \mathcal{F}, \sup _{V \geq V_{0}}\left\|\pi_{\varphi_{V}}(A)\right\|<\infty$ for all $A \in \mathcal{A}_{V_{0}}$.

Proof. (1) Let $A \in \mathcal{A}$; hence $A \in \mathcal{A}_{V_{0}}$ for some $V_{0}$. Let $X \in \mathfrak{N}_{\omega}$. Choose $V$ so that $A \in \mathcal{A}_{V}$ and $X \in \mathcal{A}_{V}$. Then

$$
\begin{aligned}
\omega_{V}\left(X^{\dagger} A^{\dagger} A X\right) & =\varphi_{V}\left(X^{\dagger} A^{\dagger} A X\right) \\
& \leq\left\|\pi_{\varphi_{V}}(A)\right\|^{2} \varphi_{V}\left(X^{\dagger} X\right) \\
& \leq \sup _{V^{\prime} \geq V_{0}}\left\|\pi_{\varphi_{V^{\prime}}}(A)\right\|^{2} \omega_{V}\left(X^{\dagger} X\right),
\end{aligned}
$$


showing that $\omega$ is admissible, and, for any $V \in \mathcal{F}$ and $A \in \mathcal{A}_{V}$,

$$
\left\|\pi_{\omega}(A)\right\| \leq \sup _{V^{\prime} \geq V}\left\|\pi_{\varphi_{V^{\prime}}}(A)\right\| .
$$

(2) Let $V \in \mathcal{F}$ be fixed. Let $X \in \mathfrak{N}_{\omega} \cap \mathcal{A}_{V}$. Then

$$
\varphi_{V}\left(X^{\dagger} X\right)=\omega_{V}\left(X^{\dagger} X\right)=\operatorname{tr}\left(\rho_{V} X^{\dagger} X\right) \leq \operatorname{Lim}_{V \rightarrow \infty} \operatorname{tr}\left(\rho_{V} X^{\dagger} X\right)=\omega\left(X^{\dagger} X\right) .
$$

Suppose $\omega$ is admissible. By Lemma $5.3 \varphi_{V}$ is admissible, and $\left\|\pi_{\varphi_{V}}(A)\right\| \leq$ $\left\|\pi_{\omega}(A)\right\|\left(A \in \mathcal{A}_{V}\right)$. Thus $\sup _{V^{\prime} \geq V}\left\|\pi_{\varphi_{V^{\prime}}}(A)\right\| \leq\left\|\pi_{\omega}(A)\right\|\left(A \in \mathcal{A}_{V}\right)$. This completes the proof.

Remark. The equilibrium state for (i) the BCS-Bogolubov model of superconductivity, as well as for (iii) interacting Boson models, can be described by quasi-weights of form $\varphi_{\Omega}$ [3], and using Proposition 6.2.4, it follows that these quasi-weights are strictly inadmissible.

6.3. Vector (quasi-) weights defined by $*$-representations. Let $(\pi, \mathcal{D}(\pi), \mathcal{H})$ be a closed $*$-representation of a $*$-algebra $\mathcal{A}$. Let $\xi \in \mathcal{H}$. This defines a quasiweight $f_{\xi}$ as follows:

$$
\begin{aligned}
& \mathfrak{N}_{f_{\xi}}=\left\{x \in \mathcal{A}: \xi \in \mathcal{D}\left(\pi\left(x^{*}\right)^{*}\right), \pi\left(x^{*}\right)^{*} \xi \in \mathcal{D}(\pi)\right\}, \\
& f_{\xi}\left(x^{*} x\right)=\left\|\pi\left(x^{*}\right)^{*} \xi\right\|^{2}, \quad x \in \mathfrak{N}_{f_{\xi}} .
\end{aligned}
$$

Then $f_{\xi}$ is a quasi-weight on $\mathcal{P}\left(\mathfrak{N}_{f_{\xi}}\right)$ in $\mathcal{A}$ and the GNS-representation $\pi_{f_{\xi}}$ for $f_{\xi}$ is unitarily equivalent to the closure of $\pi\left\lceil\pi\left(\mathfrak{N}_{f_{\xi}}^{*}\right)^{*} \xi\right.$. In particular, if $\pi\left(\mathfrak{N}_{f_{\xi}}^{*}\right)^{*} \xi$ is dense in $\left(\mathcal{D}(\pi), t_{\pi}\right), \pi_{f_{\xi}}$ is unitarily equivalent to $\pi$ (denoted by $\pi_{f_{\xi}} \cong \pi$ ), and so $f_{\xi}$ is admissible iff $\pi$ is a bounded operator representation. We investigate the (approximately) admissibility and the strictly unadmissibility of $f_{\xi}$ in the following cases:

(A) Let $\mathcal{A}$ be the $*$-algebra consisting of polynomially dominated measurable function on $\mathbb{R}_{+}$, i.e., $f \in \mathcal{A}$ iff $f: \mathbb{R}_{+} \longrightarrow \mathbb{C}$ is a measurable function for which there exists a polynomial $p$ such that $|f(t)| \leq p(t), \forall t \in \mathbb{R}_{+}$. Let $H$ be a positive self-adjoint operator in a Hilbert space $\mathcal{H}$. Let $(\pi, \mathcal{D}(\pi), \mathcal{H})$ be the $*$-representation of $\mathcal{A}$, defined as $\mathcal{D}(\pi)=C^{\infty}(H)=\bigcap_{n=1}^{\infty} \mathcal{D}\left(H^{n}\right), \pi(f)=f(H)\left\lceil_{C^{\infty}(H)}\right.$. As noted in [14], $\pi$ is a self-adjoint representation of $\mathcal{A}$, and $\pi\left(\mathfrak{N}_{f_{\xi}}^{*}\right)^{*} \xi$ is dense in $\left(C^{\infty}(H), t_{\pi}\right)$ by a consequence of the spectral theorem. Thus $\pi \cong \pi_{f_{\xi}}$ and $f_{\xi}$ is admissible iff $H$ is a bounded operator. Suppose that $f_{\xi}$ is regular. Now the self-adjointness of $H$ implies that $C^{\infty}(H)$ contains a dense set of bounded vectors for $H$. Now each bounded vector for $H$ is a bounded vector for $f(H), f \in \mathcal{A}$. It follows that when $H$ is unbounded, $f_{\xi}$ is an approximately admissible, non-admissible quasi-weight.

(B) Let $\mathcal{A}$ be the unbounded CCR algebra of one degree of freedom. $\mathcal{A}$ is the *-algebra of polynomials generated by Hermitian generators $p$ and $q$ satisfying $p q-$ $q p=-i 1$. Let $\pi_{0}$ be the Schrödinger representation of $\mathcal{A}$ defined on $L^{2}(\mathbb{R})$ having the domain $\mathcal{D}(\pi)=S(\mathbb{R})$. (Schwartz space of rapidly decreasing $C^{\infty}$-functions on $\mathbb{R})$ as $\pi_{0}(p) f=-i \frac{d f}{d t},\left(\pi_{0}(q) f\right)(t)=t f(t)$. Then $\pi_{0}$ is self-adjoint, faithful and irreducible (i.e. $\left.\pi_{0}(\mathcal{A})_{\mathrm{w}}^{\prime}=\mathbb{C} I\right)$.

Now let $\left(f_{n}\right)$ be normalized Hermite functions in $S(\mathbb{R})$. The number operator $N$ having domain $\mathcal{D}(N)=S(\mathbb{R})$ is defined as $N=\sum_{n=0}^{\infty}(n+1) f_{n} \otimes \overline{f_{n}}$. Let $\mathcal{K}$ be the *-algebra of polynomially dominated continuous function on $\mathbb{R}_{+}$viz. $f \in C\left(\mathbb{R}_{+}\right)$ and there exists a polynomial $p$ such that $|f(t)| \leq p(t), \forall t$. Let $\mathcal{B}$ be the $*$-algebra generated by $\mathcal{A}$ and $\mathcal{K}$. Define a representation $\pi$ of $\mathcal{B}$ having domain $S(\mathbb{R})$ in the 
Hilbert space $L^{2}(\mathbb{R})$ by $\pi(z)=\pi_{0}(z)$ if $z \in \mathcal{A}, \pi(z)=f(z)$ if $z=f \in \mathcal{K}$. Then $\pi$ is self-adjoint and $\pi\left(\mathfrak{N}_{f_{\xi}^{*}}^{*}\right) \xi$ is dense in $S(\mathbb{R})$. Thus $\pi_{f_{\xi}} \cong \pi$. Since $\pi$ is not bounded and irreducible, it follows from Lemma 5.2 that $f_{\xi}$ is strictly inadmissible for each non-zero $\xi \in L^{2}(\mathbb{R})$.

6.4. Point evaluations. Let $X$ be a non-compact completely regular Hausdorff space. Let $C(X)$ be the algebra of all continuous complex functions on $X$. Let $C_{b}(X)=\{f \in C(X): f$ is bounded $\}$. All non-zero multiplicative functionals on $C(X)$ (resp. on $C_{b}(X)$ ) are given, via point evaluations, by points of the Hewitt real compactification $\nu X$ (resp. the Stone-Cech compactification $\beta X$ ). Let $\mathcal{A}$ be a *-subalgebra of $C(X)$ containing $C_{b}(X)$. Let $x \in \beta X \backslash \nu X$. Given $f \in \mathcal{A}, f \geq 0$, there exist $f_{n} \geq 0$ in $C_{b}(X)$ such that $f_{n} \uparrow f$. Then $\varphi(f)=\lim _{n} f_{n}(x)=\sup _{n} f_{n}(x)$ defines a weight in $\mathcal{A}$. It is easy to check that $\pi_{\varphi}$ is the 1 -dimensional representation defined by the evaluation $\widehat{x}$ at $x$. Thus $\varphi$ is admissible.

6.5. Weights on smooth subalgebras of a $\mathbf{C}^{*}$-algebra. Let $\mathcal{A}$ be a $\mathrm{C}^{*}$-algebra with identity 1 . A $*$-subalgebra $\mathcal{B}$ of $\mathcal{A}$ with $1 \in \mathcal{B}$ is smooth if it satisfies the following:

(i) $\mathcal{B}$ is a complete locally convex $*$-algebra with a topology $t$.

(ii) $\mathcal{B}$ is dense in $\mathcal{A}$ and the induction $(\mathcal{B}, t) \longrightarrow(A,\|\cdot\|)$ is continuous.

(iii) $\mathcal{B}$ is spectrally invariant in $\mathcal{A}$.

Proposition 6.5.1. Let $\mathcal{B}$ be a smooth subalgebra of a $C^{*}$-algebra $\mathcal{A}$. Let $\varphi$ be a quasi-weight on $P\left(\mathfrak{N}_{\varphi}\right)$ in $\mathcal{B}$. The following statements hold:

(i) $\varphi$ is admissible, and the bounded $*$-representation $\pi_{\varphi}$ of $\mathcal{B}$ can be uniquely extended to a bounded $*$-representation $\widetilde{\pi_{\varphi}}$ of $\mathcal{A}$ on $\mathcal{H}_{\varphi}$.

(ii) If $\dot{\varphi}$ is continous on $\mathcal{D}(\varphi), \varphi$ can be extended to a quasi-weight $\widetilde{\varphi}$ on $\mathcal{P}\left(\overline{\mathfrak{N}_{\varphi}}\right)$, where $\overline{\mathfrak{N}}_{\varphi}$ is the closure of $\mathfrak{N}_{\varphi}$ in $(\mathcal{A},\|\cdot\|)$.

Proof. For any $x \in \mathcal{B}, \operatorname{sp}_{\mathcal{A}}(x)=\operatorname{sp}_{\mathcal{B}}(x)$. This gives the spectral radius $r(x) \leq\|x\|$, showing that $(\mathcal{B},\|\cdot\|)$ and hence $(\mathcal{B}, t)$ are Q-algebras. Let $\varphi$ be a quasi-weight on $P\left(\mathfrak{N}_{\varphi}\right)$ in $\mathcal{B}$. By Theorems 4.2 and $3.12, \varphi$ is admissible and the GNS-representation $\pi_{\varphi}$ is a bounded operator representation. Since $(\mathcal{B},\|\cdot\|)$ is a Q-algebra, $\pi_{\varphi}:(\mathcal{B},\|\cdot\|)$ $\longrightarrow B\left(\mathcal{H}_{\varphi}\right)$ is continuous, hence extends to a $*$-homomorphism $\widetilde{\pi_{\varphi}}:(\mathcal{A},\|\cdot\|) \longrightarrow$ $B\left(\mathcal{H}_{\varphi}\right)$. Assume that $\dot{\varphi}$ is continuous on $\mathcal{D}(\varphi)$. Now, $\mathfrak{N}_{\varphi}$ being a proper left ideal of $\mathcal{B}$, it contains no invertible element of $\mathcal{B}$. As $\mathcal{B}$ is inverse closed in its completion $\mathcal{A}$, $\overline{\mathfrak{N}_{\varphi}}$ contains no invertible element of $\mathcal{A}$. Thus $1 \notin \overline{\mathfrak{N}_{\varphi}}$ and $\overline{\mathfrak{N}_{\varphi}}$ is a proper left ideal of $\mathcal{A}$. Let $x \in \overline{\mathfrak{N}_{\varphi}}$. Then $x^{*} x \in \mathcal{P}\left(\overline{\mathfrak{N}_{\varphi}}\right)$, and there exists a sequence $\left\{x_{n}\right\}$ in $\mathfrak{N}_{\varphi}$ such that $x_{n} \rightarrow x$. Then $x_{n}^{*} x_{n} \in \mathfrak{N}_{\varphi}{ }^{*} \mathfrak{N}_{\varphi}$ and $x_{n}^{*} x_{n} \rightarrow x^{*} x$. Thus $\left\{x_{n}^{*} x_{n}\right\}$ is $\|\cdot\|-$ bounded; hence by assumption the sequence $\left\{\varphi\left(x_{n}^{*} x_{n}\right)\right\} \in l^{\infty}$. Let $\omega$ denote any positive linear functional on $l^{\infty}$ vanishing on $C_{0} \equiv\left\{\left\{\alpha_{n}\right\} \subset \mathbb{C}: \operatorname{Lim}_{n \rightarrow \infty} \alpha_{n}=0\right\}$ and $\omega(\{\alpha\})=\alpha(\alpha \in \mathbb{C})$. Define $\widetilde{\varphi}: \mathcal{P}\left(\overline{\mathfrak{N}_{\varphi}}\right) \rightarrow[0, \infty)$ by $\widetilde{\varphi}\left(x^{*} x\right)=\omega\left(\left\{\varphi\left(x_{n}^{*} x_{n}\right)\right\}\right)$. Note that $\widetilde{\varphi}$ is well-defined. Indeed, let there be sequences $\left\{x_{n}\right\}$ and $\left\{y_{n}\right\}$ in $\mathfrak{N}_{\varphi}$ such that $x_{n} \rightarrow x$ and $y_{n} \rightarrow x$. Then $a_{n} \equiv y_{n}^{*} y_{n}-x_{n}^{*} x_{n} \in \mathcal{D}(\varphi)$ and $a_{n} \rightarrow 0$. By the assumption $\dot{\varphi}\left(a_{n}\right) \rightarrow 0$; hence $\omega\left(\left\{\dot{\varphi}\left(a_{n}\right)\right\}\right)=0$. Then $\varphi\left(y_{n}^{*} y_{n}\right)=\dot{\varphi}\left(a_{n}\right)+\varphi\left(x_{n}^{*} x_{n}\right)$ gives $\omega\left(\left\{\varphi\left(y_{n}^{*} y_{n}\right)\right\}\right)=\omega\left(\left\{\varphi\left(x_{n}^{*} x_{n}\right)\right\}\right)$, which shows that $\widetilde{\varphi}$ is well-defined. Now let $z \in \mathcal{P}\left(\overline{\mathfrak{N}_{\varphi}}\right), z=\sum_{i} x_{i}^{*} x_{i}$, a finite sum with each $x_{i} \in \overline{\mathfrak{N}_{\varphi}}$. Choose $x_{i}^{(n)} \in$ $\mathfrak{N}_{\varphi}$ such that $x_{i}^{(n)} \rightarrow x_{i}$. Then $z_{n} \equiv \sum_{i} x_{i}^{(n)^{*}} x_{i}^{(n)} \in \mathcal{P}\left(\mathfrak{N}_{\varphi}\right) \rightarrow z$, and $\varphi\left(z_{n}\right)=$ $\sum_{i} \varphi\left(x_{i}^{(n)^{*}} x_{i}^{(n)}\right)$. Hence $\widetilde{\varphi}(z)=\omega\left(\left\{\varphi\left(z_{n}\right)\right\}\right)=\sum_{i} \omega\left(\left\{\varphi\left(x_{i}^{(n)^{*}} x_{i}^{(n)}\right)\right\}\right)=\sum_{i} \widetilde{\varphi}\left(x_{i}^{*} x_{i}\right)$, 
and so $\widetilde{\varphi}$ is a quasi-weight on $\mathcal{P}\left(\overline{\mathfrak{N}_{\varphi}}\right)$. Finally, let $x \in \mathfrak{N}_{\varphi}$ and $x_{n}=x, n \in \mathbb{N}$. Then $\widetilde{\varphi}\left(x^{*} x\right)=\omega\left(\left\{\varphi\left(x_{n}^{*} x_{n}\right)\right\}\right)=\varphi\left(x^{*} x\right)$, and hence $\widetilde{\varphi}$ is an extension of $\varphi$. This completes the proof.

Corollary 6.5.2. Let $\mathcal{A}$ and $\mathcal{B}$ be as above.

(1) Every positive linear functional $f$ of $\mathcal{B}$ can be uniquely extended to a positive linear functional $\widetilde{f}$ on $\mathcal{A}$. Further, if $f$ is extreme, then $\widetilde{f}$ is also extreme.

(2) Let $\mathcal{A}$ be commutative. Then the Gelfand spaces $\Delta(\mathcal{B})$ and $\Delta(\mathcal{A})$ are homeomorphic.

When $\mathcal{A}=C(M), \mathcal{B}=C^{\infty}(M)$ for a compact manifold $M$, the above recaptures the well-known facts that every positive distribution on $M$ is a measure and that $\Delta\left(C^{\infty}(M)\right)=M$.

The above proposition also applies to the Fréchet $*$-algebra $\mathcal{A}^{\infty}$ of $C^{\infty}$-elements of a $\mathrm{C}^{*}$-algebra $\mathcal{A}$ determined by an action of a Lie group $G$.

6.6. Quasi-weights in non-commutative geometry. We construct quasiweights that arise naturally in the Connes theory of non-commutative geometry and discuss their admissibility. The basic formalism of non-commutative geometry is as follows:

Let $\mathcal{A}$ be a non-commutative $*$-algebra with identity representing "a non-commutative geometric space". The algebra of non-commutative differential forms over $\mathcal{A}$ is the $*$-algebra $\Omega(\mathcal{A})$ generated by elements $a \in \mathcal{A}$, of degree 0 and elements $d a, a \in \mathcal{A}$, of degree 1 satisfying the relations $d(a+b)=d a+d b, d(\lambda a)=\lambda d a(\lambda$ a scalar $), d(a b)=a d b+(d a) b$. Then $\Omega(\mathcal{A})=\bigoplus_{n=0}^{\infty} \Omega^{n}(\mathcal{A})$ defines a gradation in $\Omega(\mathcal{A})$, where

$$
\begin{aligned}
\Omega^{0}(\mathcal{A}) & =\mathcal{A}, \\
\Omega^{n}(\mathcal{A}) & =\left\{\alpha=\sum_{j} a_{j}^{0} d a_{j}^{1} \cdots d a_{j}^{n}: a_{j}^{i} \in \mathcal{A}\right\},
\end{aligned}
$$

the vector space consisting of differential forms of degree $n$. One takes $(d a)^{*}=-d a^{*}$ and $d\left(a_{0} d a_{1} \cdots d a_{n}\right)=d a_{0} d a_{1} \cdots d a_{n}$. The differentiable structure on $\mathcal{A}$ is imposed by the presence of a $K$-cycle or a spectral triple $(\pi, \mathcal{H}, D)$ defined by the following data:

(i) $\mathcal{H}$ is a separable Hilbert space.

(ii) $\pi: \mathcal{A} \rightarrow \mathcal{B}(\mathcal{H})$ is a faithful $*$-representation of $\mathcal{A}$ into bounded operators on $\mathcal{H}$.

(iii) $D$ is a self-adjoint operator in $\mathcal{H}$ satisfying

(iii) ${ }_{1}\{a \in \mathcal{A}:[D, \pi(a)]$ is bounded $\}=\mathcal{A}$,

(iii) $)_{2} D$ has a compact resolvent so that for all $\lambda \notin s p D,(\lambda I-D)^{-1}$ is a compact operator.

Now the representation $\pi$ of $\mathcal{A}$ can be extended as a $*$-representation $\pi: \Omega(\mathcal{A}) \rightarrow$ $\mathcal{B}(\mathcal{H})$ by

$$
\pi\left(a^{0} d a^{1} \cdots d a^{n}\right)=\pi\left(a^{0}\right)\left[D, \pi\left(a^{1}\right)\right]\left[D, \pi\left(a^{2}\right)\right] \cdots\left[D, \pi\left(a^{n}\right)\right] .
$$

Now let $\mathcal{K}(\mathcal{H})$ denote the ideal of compact operators. For $T \in \mathcal{K}(\mathcal{H})$, let $|T|=$ $\left(T^{*} T\right)^{\frac{1}{2}}$. Let $\left\{\xi_{n}\right\}$ be an orthonormal basis in $\mathcal{H}$. Let $\left\{\mu_{n}(T)\right\}$ denote the eigenvalues of $|T|$ arranged in decreasing order, counted according to multiplicities. Let 
$\sigma_{N}(T)=\sum_{n=0}^{N-1} \mu_{n}(T)$. Then $\mu_{n}(T) \rightarrow 0$, which motivates calling compact operators the non-commutative infinitesimals (in representation in $\mathcal{B}(\mathcal{H})$ ). Then infinitesimals of order $\alpha$ constitute the two sided ideal

$$
\mathcal{K}_{\alpha}(\mathcal{H})=\left\{T \in \mathcal{B}(\mathcal{H}): \mu_{n}(T)=O\left(n^{-\alpha}\right) \text { as } n \rightarrow \infty\right\}
$$

in $\mathcal{B}(\mathcal{H})$.

(A) Dixmier trace. Clearly, we have

$$
\begin{aligned}
C^{1+}(\mathcal{H}) & \equiv\left\{T \in \mathcal{K}(\mathcal{H}): \sigma_{N}(T)=O(\log N) \text { as } N \rightarrow \infty\right\} \\
& \supset \mathcal{K}_{1}(\mathcal{H})=\left\{T \in \mathcal{K}(\mathcal{H}): \mu_{n}(T)=O\left(\frac{1}{n}\right) \text { as } n \rightarrow \infty\right\} \\
& \left.\supset \mathcal{K}_{1+}(\mathcal{H}) \text { (infinitesimals of order }>1\right) \\
& \equiv\left\{T \in \mathcal{K}(\mathcal{H}): \mu_{n}(T)=o\left(\frac{1}{n}\right) \text { as } n \rightarrow \infty\right\} \\
& =\left\{T \in \mathcal{K}(\mathcal{H}): \exists \varepsilon>0 \text { s.t. } \mu_{n}(T)=O\left(\frac{1}{n^{1+\varepsilon}}\right) \text { as } n \rightarrow \infty\right\} \\
& \subset C^{1}(\mathcal{H}) \equiv\left\{T \in \mathcal{K}(\mathcal{H}) ; \sum_{n=0}^{\infty} \mu_{n}(T)<\infty\right\}
\end{aligned}
$$

(trace class operators).

Let $\omega$ be the generalized limit (Banach limit) on $l^{\infty}(\mathbb{N})$, that is, a translation invariant positive linear functional on $l^{\infty}(\mathbb{N})$ vanishing on $C_{0}(\mathbb{N})$. For $T \geq 0$ in $C^{1^{+}}(\mathcal{H})$, let

$$
\begin{aligned}
\operatorname{Tr}_{\omega}(T) & \equiv \omega\left(\left\{\frac{\sigma_{N}(T)}{\log N}\right\}\right) \\
& \equiv \tau_{\omega}(T) \\
& \equiv \operatorname{Lim}_{N \rightarrow \infty} \frac{\sigma_{N}(T)}{\log N}
\end{aligned}
$$

( $\operatorname{Lim}_{\omega}$ indicates that the limit is defined in some kind of Cesáro mean). Then $\operatorname{Tr}_{\omega}$ defines a tracial linear functional on $C^{1^{+}}(\mathcal{H})$ vanishing on $\mathcal{K}_{1^{+}}(\mathcal{H})$ which extends to $\mathcal{B}(\mathcal{H})$ as a trace by taking $\sigma_{N}(T)=\sum_{n=0}^{N}\left\langle|T| \xi_{n}, \xi_{n}\right\rangle$. This $\operatorname{Tr}_{\omega}$ is called the Dixmier trace. Now we define a quasi-weight $\left(\tau_{\omega}, \mathfrak{N}_{\tau_{\omega}}\right)$ in $\Omega(\mathcal{A})$ by taking

$$
\left\{\begin{array}{l}
\mathfrak{N}_{\tau_{\omega}}=\left\{a \in \Omega(\mathcal{A}): \pi(a) \in C^{1+}(\mathcal{H})\right\} \\
\tau_{\omega}\left(a^{*} a\right)=\operatorname{Tr}_{\omega}\left(\pi(a)^{*} \pi(a)\right), a \in \mathfrak{N}_{\tau_{\omega}}
\end{array}\right.
$$

Proposition 6.6.1. $\tau_{\omega}$ is an admissible quasi-weight in $\Omega(\mathcal{A})$, and if $a \in \Omega(\mathcal{A})$ is such that $\pi(a)$ is compact and measurable, then

$$
\begin{aligned}
\tau_{\omega}(a)= & \lim _{\lambda \rightarrow \infty} \frac{1}{\log \lambda} \int_{0}^{\lambda} \frac{d u}{u} \frac{1}{\log u} \\
& \cdot \inf \left\{\|X\|_{1}+u\|Y\| ; \begin{array}{l}
X \in C^{1}(\mathcal{H}), Y \in \mathcal{B}(\mathcal{H}) \\
X+Y=\pi(a)
\end{array}\right\},
\end{aligned}
$$

where $\|X\|_{1}=\operatorname{tr}(|X|)$.

The following expression for $\operatorname{Tr}_{\omega}$ is discussed in [10]. For $T \in K(\mathcal{H}), \lambda>0$, let

$$
\sigma_{\lambda}(T)=\inf \left\{\|X\|_{1}+\lambda\|Y\|: X \in C^{1}(\mathcal{H}), Y \in \mathcal{B}(\mathcal{H}) \text { with } X+Y=T\right\} .
$$


For $\lambda \in \mathbb{N}, \sigma_{\lambda}(T)$ coincides with $\sum_{n=0}^{N-1} \mu_{n}(T)$. Let

$$
\tau_{\lambda}(T)=\frac{1}{\log \lambda} \int_{0}^{\lambda} \frac{\sigma_{u}(T)}{\log u} \frac{d u}{u} .
$$

Then $\operatorname{Tr}_{\omega}(T)=\lim _{\lambda \rightarrow \infty} \tau_{\lambda}(T)$ (=any limit point of $\tau_{\lambda}(T)$ ), and $T$ is measurable if $\tau_{\lambda}(T)$ is convergent when $\lambda \rightarrow \infty$. In this case, $\operatorname{Tr}_{\omega}(T)=\lim _{\lambda \rightarrow \infty} \tau_{\lambda}(T)$ independently of $\omega$.

Proof. Let $a \in \Omega(\mathcal{A}), x \in \mathfrak{N}_{\tau_{\omega}}$. Then

$$
\begin{aligned}
\tau_{\omega}\left(x^{*} a^{*} a x\right) & =\operatorname{Tr}_{\omega}\left(\pi(x)^{*} \pi(a)^{*} \pi(a) \pi(x)\right) \\
& =\operatorname{Tr}_{\omega}\left(\pi(a)^{*} \pi(a) \pi(x) \pi(x)^{*}\right) \\
& \leq\|\pi(a)\|^{2} \operatorname{Tr}_{\omega}\left(\pi(x) \pi(x)^{*}\right) \quad \text { (by [9]) } \\
& =\|\pi(a)\|^{2} \operatorname{Tr}_{\omega}\left(\pi(x)^{*} \pi(x)\right) \\
& =\|\pi(a)\|^{2} \tau_{\omega}\left(x^{*} x\right) .
\end{aligned}
$$

The remaining part follows from [10, p.6212].

Remark 6.6.2. Let $\mathcal{M}$ be an $\mathrm{O}^{*}$-algebra on a dense subspace $\mathcal{D}$ in a Hilbert space $\mathcal{H}$. Then a quasi-weight $\left(\varphi, \mathfrak{N}_{\varphi}\right)$ is defined in $\mathcal{M}$ by taking

$$
\left\{\begin{array}{l}
\mathfrak{N}_{\varphi}=\left\{X \in \mathcal{M}: \overline{\left(X^{\dagger} A^{\dagger} A X\right)} \in C^{1+}(\mathcal{H}) \text { for all } A \in \mathcal{M}\right\} \\
\varphi\left(X^{\dagger} X\right)=\operatorname{Tr}_{\omega}\left(X^{\dagger} X\right), X \in \mathfrak{N}_{\varphi} .
\end{array}\right.
$$

(B) The d-dimensional volume integral. Following [10, p.6219], the spectral triple $(\pi, \mathcal{H}, D)$ is of dimension $d$ if $|D|^{-1}$ is an infinitesimal of order $\frac{1}{d}$. In this case, the sequence $\left\{\sigma_{n}\left(|D|^{-d}\right) / \log n\right\}$ is bounded, and the $\mathcal{K}$-cycle is also called $d^{+}$summable. For a $d^{+}$-summable cycle, the quantity $\varphi(a) \equiv \operatorname{Tr}_{\omega}\left(|D|^{-d} \psi(a)\right), a \in$ $\Omega(\mathcal{A})$, defines a positive linear functional on $\Omega(\mathcal{A})$ whose restriction to $\mathcal{A}$ is a trace $\left[9\right.$, Theorem 1.3]. Following [10, p.6219], $\varphi \Gamma_{\mathcal{A}}$ is an analogue of the volume integral, denoted by

$$
\varphi(a)=\operatorname{Tr}_{\omega}\left(\pi(a)|D|^{-d}\right)=\operatorname{Tr}_{\omega}\left(|D|^{-d} \pi(a)\right)
$$

In the general case, this defines a quasi-weight in $\mathcal{A}$ as follows:

$$
\begin{aligned}
\mathfrak{N}_{\varphi} & \equiv\left\{x \in \mathcal{A}: \operatorname{Tr}_{\omega}\left(|D|^{-d} \pi\left(x^{*} a^{*} a x\right)\right)<\infty \text { for each } a \in \mathcal{A}\right\} \\
& \left.=\left\{x \in \mathcal{A}:|D|^{-d} \pi\left(x^{*} a^{*} a x\right)\right) \in C^{1+}(\mathcal{H}) \text { for each } a \in \mathcal{A}\right\} \\
\varphi(x) & \equiv \operatorname{Tr}_{\omega}\left(|D|^{-d} \pi(x)\right) \\
& =\operatorname{Tr}_{\omega}\left(\pi(x)|D|^{-d}\right) \\
& =\omega\left(\left\{\sigma_{n}\left(|D|^{-d} \pi(x)\right) / \log n\right\}\right) \\
& =\omega\left(\left\{\sum_{k=0}^{n-1}\left\|\pi(x)|D|^{-\frac{d}{2}} \xi_{k}\right\|^{2} / \log n\right\}\right)
\end{aligned}
$$


Now let $a \in \mathcal{A}, x \in \mathfrak{N}_{\varphi}$. Then

$$
\begin{aligned}
\varphi\left(x^{*} a^{*} a x\right) & =\operatorname{Tr}_{\omega}\left(\pi\left(x^{*} a^{*} a x\right)|D|^{-d}\right) \\
& =\operatorname{Tr}_{\omega}\left(\pi\left(a^{*} a\right) \pi(x)|D|^{-\frac{d}{2}}|D|^{-\frac{d}{2}} \pi\left(x^{*}\right)\right) \quad(\text { by }[9, \mathrm{p} .182]) \\
& =\operatorname{Tr}_{\omega}\left(\left.\left.|\pi(a)|^{2}|| D\right|^{-\frac{d}{2}} \pi(x)^{*}\right|^{2}\right) \\
& \leq\|\pi(a)\|^{2} \operatorname{Tr}_{\omega}\left(\left.\left.|| D\right|^{-\frac{d}{2}} \pi(x)^{*}\right|^{2}\right) \quad(\text { by }[9, \mathrm{p} .184]) \\
& =\|\pi(a)\|^{2} \operatorname{Tr}_{\omega}\left(\pi\left(x^{*} x\right)|D|^{-d}\right) \\
& =\|\pi(a)\|^{2} \varphi\left(x^{*} x\right) .
\end{aligned}
$$

Hence we have the following.

Proposition 6.6.3. The d-dimensional volume integral $\varphi$ on $\mathcal{A}$ defines an admissible quasi-weight. Further, $\varphi$ is finite if and only if the $K$-cycle is $d^{+}$-summable.

Remark 6.6.4. The formula

$$
\varphi\left(a_{0} d a_{1} \cdots d a_{n}\right)=i^{n} \operatorname{Tr}_{\omega}\left(|D|^{-d} \pi\left(a_{0}\right)\left[D, \pi\left(a_{1}\right)\right]\left[D, \pi\left(a_{2}\right)\right] \cdots\left[D, \pi\left(a_{n}\right)\right]\right)
$$

defines a quasi-weight in $\Omega(\mathcal{A})$. We do not know whether it is admissible or not. The hypertrace property of $X \rightarrow \operatorname{Tr}_{\omega}\left(|D|^{-d} X\right)$ on $\pi(\mathcal{A})$ used above is no longer available on $\pi(\Omega(\mathcal{A}))$ [9, p.184].

(C) The infinite dimensional case. Motivated by [8, p.6259], consider the quasiweight $\left(\psi, \mathfrak{N}_{\psi}\right)$ in $\Omega(\mathcal{A})$ defined by the following data:

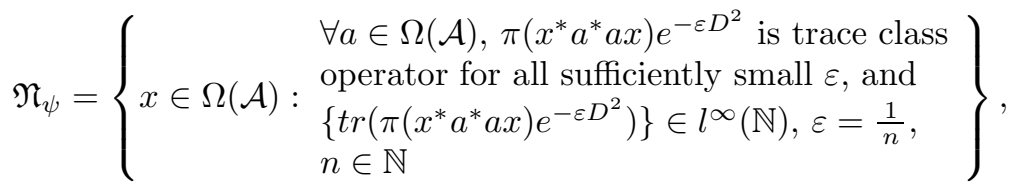

$$
\begin{aligned}
& \psi\left(x^{*} x\right) \equiv \omega\left(\left\{\operatorname{tr}\left(\pi\left(x^{*} x\right) e^{-\varepsilon D^{2}}\right)\right\}_{\varepsilon}\right) \\
& =\operatorname{Lim}_{\substack{\omega \\
\varepsilon \rightarrow 0}} \operatorname{tr}\left(\pi\left(x^{*} x\right) e^{-\varepsilon D^{2}}\right) \\
& =\operatorname{Lim}_{\varepsilon \rightarrow 0} \sum_{n=1}^{\infty}\left\|\pi(x) e^{-\frac{\varepsilon}{2} D^{2}} \xi_{n}\right\|^{2} \\
& =\underset{\substack{\omega \\
\varepsilon \rightarrow 0}}{\operatorname{Lim}}\left\|\pi(x) e^{-\frac{\varepsilon}{2} D^{2}}\right\|_{2}{ }^{2}, \quad x \in \mathfrak{N}_{\psi},
\end{aligned}
$$

where $\operatorname{Lim}_{\varepsilon \rightarrow 0}$ indicates the limit in suitable Cesáro mean and $t r$ denotes the usual trace in $\stackrel{\varepsilon \rightarrow 0}{\mathcal{B}}(\mathcal{H})$.

Proposition 6.6.5. The quasi-weight $\psi$ in $\Omega(\mathcal{A})$ is admissible. Further, $\psi$ is finite if and only if the $K$-cycle is $\theta$-summable.

Recall that the $K$-cycle $(\pi, \mathcal{H}, \mathcal{D})$ is $\theta$-summable if $e^{-\varepsilon D^{2}}$ is a trace class operator for all $\varepsilon>0$. This $\theta$-summability provides a tractable class of infinite dimmensional 
$K$-cycles, being a kind of compactness condition [8, p. 6258]; and it defines an integral on $\Omega(\mathcal{A})$ as

$$
f_{\alpha}=\operatorname{Lim}_{\varepsilon \rightarrow 0} \frac{\operatorname{tr}\left(\pi(\alpha) e^{-\varepsilon D^{2}}\right)}{\operatorname{tr}\left(e^{-\varepsilon D^{2}}\right)} .
$$

Proof. Let $x \in \mathfrak{N}_{\varphi}, a \in \Omega(\mathcal{A})$. Then, for any $\varepsilon>0$,

$$
\begin{aligned}
\operatorname{tr}\left(\pi\left(x^{*} a^{*} a x\right) e^{-\varepsilon D^{2}}\right) & =\left\|\pi(a x) e^{-\frac{\varepsilon}{2} D^{2}}\right\|_{2}{ }^{2} \\
& \leq\|\pi(a)\|^{2}\left\|\pi(x) e^{-\frac{\varepsilon}{2} D^{2}}\right\|_{2}{ }^{2} \\
& =\|\pi(a)\|^{2} \operatorname{tr}\left(\pi\left(x^{*} x\right) e^{-\varepsilon D^{2}}\right) .
\end{aligned}
$$

The sequences $\left\{\delta_{\varepsilon}=\operatorname{tr}\left(\pi\left(x^{*} a^{*} a x\right) e^{-\varepsilon D^{2}}\right)\right\}_{\varepsilon}$ and $\left\{\eta_{\varepsilon}=\operatorname{tr}\left(\pi\left(x^{*} x\right) e^{-\varepsilon D^{2}}\right)\right\}_{\varepsilon}$ are in $l^{\infty}$, and $0 \leq \delta_{\varepsilon} \leq\|\pi(a)\|^{2} \eta_{\varepsilon}$. Now, $\omega$ being a positive linear functional on $l^{\infty}$, it follows that $\omega\left(\left\{\delta_{\varepsilon}\right\}\right) \leq\|\pi(a)\|^{2} \omega\left(\left\{\eta_{\varepsilon}\right\}\right)$. This gives

$$
\psi\left(x^{*} a^{*} a x\right) \leq\|\pi(a)\|^{2} \psi\left(x^{*} x\right) .
$$

(D) The integral $f f(a)|d a|^{p}(p>0)$. Let $a \in \mathcal{A}$ identify with $\pi(a)$, assumed to be a self-adjoint operator in $\mathcal{B}(\mathcal{H})$. Let $F=D|D|^{-1}$. Following [10, p. 6219], $d a \equiv[F, a]$ is a compact operator by the definition of spectral triple. Then $|d a|^{p}=|[F, a]|^{p}$ makes sense in $\mathcal{B}(\mathcal{H})$. The spectral theorem defines $f(a)$ in $\mathcal{B}(\mathcal{H})$ for each $f \in C_{0}(\mathbb{R})$. The integral $f f(a)|d a|^{p}=\operatorname{Tr}_{\omega}\left(f(a)|d a|^{p}\right)$ defines a quasi-weight $\varphi$ in $C_{0}(\mathbb{R})$ as follows:

$$
\begin{aligned}
\mathfrak{N}_{\varphi} & =\left\{f \in C_{0}(\mathbb{R}): f(a)|d a|^{p} \in C^{1+}(\mathcal{H})\right\} \\
\varphi(f) & =f f(a)|d a|^{p}=\operatorname{Tr}_{\omega}\left(f(a)|d a|^{p}\right), f \geq 0 \text { in } \mathfrak{N}_{\varphi} .
\end{aligned}
$$

Since $a$ is a bounded operator, $\varphi$ turns out to be an admissible quasi-weight in $C_{0}(\mathbb{R})$.

\section{ACKNOWLEDGEMENT}

This work was done when S. J. Bhatt visited the Department of Applied Mathematics at Fukuoka University during September and October 1995. He is grateful to Professor Atsushi Inoue for the invitation and warm hospitality, and to colleagues in the Applied Mathematics Department and to Fukuoka University for making the visit possible. He is thankful to Sardar Patel University for granting leave.

\section{REFERENCES}

1. G. R. Allan, A spectral theory for locally convex algebras, Proc. London Math. Soc., 15 (1965), 399-421. MR 31:619

2. G. R. Allan, On a class of locally convex algebras, Proc. London Math. Soc., 17 (1967), 91-114. MR 34:4937

3. J. -P. Antoine, H. Ogi, A. Inoue and C. Trapani, Standard generalized vectors on the space of Hilbert-Schmidt operators, Ann. Inst. H. Poincaré, 63 (1995), 177-210. MR 96i:47082

4. R. Arens, The space $L^{\omega}$ and convex topological rings, Bull. Amer. Math. Soc., 52 (1946), 931-935. MR 8:165d

5. B. A. Barnes, The properties *-regularity and uniqueness of $C^{*}$-norm in a general $*$-algebra, Trans. Amer. Math. Soc., 279 (1983), 841-859. MR 85f: 46100 
6. S. J. Bhatt, Representability of positive functionals on abstract star algebras without identity with applications to locally convex *-algebras, Yokohama Math. J., 29 (1981), 7-16. MR 83d:46068

7. F. F. Bonsall and J. Duncan, Complete Normed Algebras, Springer-Velag, Berlin, Heidelberg, New York, 1973. MR 54:11013

8. A. F. Chamseddine, J. Fröhlich and O. Grandjean, The gravitational sector in the Connes-Lott formulation of the standard model, J. Math. Phys., 36 (1995), 6255-6275. MR 96m:81256

9. F. Cipriani, D. Guido and S. Searlatti, A remark on trace properties of K-cycles, J. Operator Theory, 35 (1996), 179-189. MR 97d:46089

10. A. Connes, Non-commutative geometry and reality, J. Math. Phys., 36 (1995), 6194-6231. MR 96g:58014

11. J. Dixmier, $C^{*}$-algebras, North Holland, 1977. MR 56:16388

12. P. G. Dixon, Generalized $B^{*}$-algebras, Proc. London Math. Soc., 21 (1970), 693-715. MR 43:3811

13. J. M. G. Fell and R. S. Doran, Representations of *-Algebras, Locally Compact Groups and Banach *-Algebraic Bundles, Vols. I, II, Academic Press, 1988. MR 90c:46001; MR 90c:46002

14. S. P. Gudder and R. L. Hudson, A noncommutative probability theory, Trans. Amer. Math. Soc., 245 (1978), 1-41. MR 81m:46092

15. A. Inoue, An unbounded generalization of the Tomita-Takezaki theory I, II, Publ. RIMS, Kyoto Univ., 22 (1986), 725-765: 23 (1987), 673-726. MR 88c:47094; MR 89d:47107

16. A. Inoue, $O^{*}$-algebras in standard system, Math. Nachr., 172 (1995), 171-190. MR 96e:47050

17. A. Inoue and W. Karwowski, Cyclic generalized vectors for algebras of unbounded operators, Publ. RIMS, Kyoto Univ., 30 (1994), 577-601. MR 96a:47081

18. A. Inoue and H. Ogi, Regular weights on algebras of unbounded operators, J. Math. Soc. Japan, 50 (1998), 227-252. CMP 98:05

19. T. W. Palmer, Banach Algebras and General Theory of $*$-Algebras, Vol I, Cambridge Univ. Press, 1994. MR 95c: 46002

20. T. W. Palmer, Spectral algebras, Rocky Mountain J. Math., 22 (1992), 293-328. MR 93d: 46079

21. N. C. Phillips, Inverse limits of $C^{*}$-algebras, J. Operator Theory, 19 (1988), 159-195. MR 90c: 46090

22. J. D. Powell, Representations of locally convex *-algebras, Proc. Amer. Math. Soc., 44 (1974), 341-346. MR 50:14248

23. R. T. Powers, Self-adjoint algebras of unbounded operators, Commun. Math. Phys., 21 (1971), 85-124. MR 44:811

24. C. E. Rickart, General Theory of Banach Algebras, Van Nostrand, 1960. MR 22:5903

25. K. Schmüdgen, Unbounded Operator Algebras and Representation Theory, Akademie-Verlag, 1990. MR 91f: 47062

26. K. Takesue, Standard representations induced by positive linear functionals, Mem. Fac. Sci. Kyushu Univ., 37 (1983), 211-225. MR 85j:46096

Department of Mathematics, Sardar Patel University, Vallabh Vidyanagar 388120 , Gujarat, India

Department of Applied Mathematics, Fukuoka University, Nanakuma, Jonan-ku, FUKUOKA, 814-80 JAPAN

E-mail address, Inoue: sm010888@ssat.fukuoka-u.ac.jp

E-mail address, Ogi: sm037255@ssat.fukuoka-u.ac.jp 